OCIP/C 93-18

UQAM-PHE-9306

hep-ph/9401306

December 1993

\title{
A PHENOMENOLOGICAL STUDY OF THE PROCESS
}

\section{$e^{+} e^{-} \rightarrow \mu^{+} \mu^{-} \nu_{l} \bar{\nu}_{l}$ AT HIGH ENERGY $e^{+} e^{-}$COLLIDERS \\ AND MEASUREMENT OF THE $Z W W$ AND $\gamma W W$ COUPLINGS}

\author{
Gilles Couture* \\ Département de Physique, Université du Québec à Montréal \\ C.P. 8888, Succ. A, Montréal, Québec, Canada, H3C 3P8 \\ Stephen Godfrey ${ }^{\dagger}$ \\ Ottawa-Carleton Institute for Physics \\ Department of Physics, Carleton University, Ottawa CANADA, K1S 5B6
}

\begin{abstract}
We perform a detailed study of the process $e^{+} e^{-} \rightarrow \mu^{+} \mu^{-} \nu_{l} \bar{\nu}_{l}$ including all contributions. The contributions other than from real gauge boson production leads to a rich phenomenology. We explore the use of the process as a means of precision measurement of the $Z W W$ and $\gamma W W$ vertices. We concentrate on LEP II energies, $\sqrt{s}=200 \mathrm{GeV}$, and energies appropriate to the proposed Next Linear Collider (NLC) high energy $e^{+} e^{-}$collider with center of mass energies $\sqrt{s}=500$ and 1 TeV. At 200 $\mathrm{GeV}$, the process offers, at best, a consistency check of other processes being considered at LEP200. At $500 \mathrm{GeV}$, the parameters $\kappa_{\gamma}, \lambda_{\gamma}, \kappa_{Z}$, and $\lambda_{Z}$ can be measured to about \pm 0.1 or better at $95 \%$ C.L. while at $1 \mathrm{TeV}$, they can be measured to about \pm 0.01 . At the high luminosities anticipated at high energy linear colliders precision measurements are likely to be limited by systematic rather than statistical errors.
\end{abstract}


PACS numbers: 12.15.Ji, 14.80.Er

Typeset Using REVTEX 


\section{INTRODUCTION}

There is a growing interest in the physics that can be studied at high energy $e^{+} e^{-}$colliders [1]. High energy $e^{+} e^{-}$colliders offer a cleaner environment than multi-TeV hadron colliders and are therefore expected to allow more quantitative studies of physics at the Fermi scale. Some of the physics topics that have been explored are precision measurement of $t$-quark properties, searches for new physics, electroweak symmetry breaking, tests of QCD, and precision measurements of the electroweak gauge bosons [2].

At the same time there is a growing appreciation that to realistically assess the physics potential of a specific process one must perform detailed studies of the final state decay products that will be observed by a detector rather than the massive, short lived states that we are directly interested in [3 8]. Performing such a study greatly increases the complexity of the analysis as one must include finite width effects of the decaying particles and all the background processes that result in the same final state. On the other hand, this complexity results in a much richer phenomenology which more closely describes what is experimentally observed. In addition, the finite width effects are, in some sense, radiative corrections of order $\Gamma / M \sim \alpha$ which must ultimately be included in a full calculation including radiative corrections [四].

In this paper we present a detailed study of the process $e^{+} e^{-} \rightarrow \nu \bar{\nu} \mu^{+} \mu^{-}$motivated by our interest in the underlying process $e^{+} e^{-} \rightarrow \nu_{e} \bar{\nu}_{e} Z^{0}$. Although this process has been studied elsewhere [9, 10], none of the previous calculations have included the decay to final state fermions with finite width effects and the nonresonant backgrounds. We find that including these contributions adds considerably to the richness of the phenomenology. We then use this process to study the $W W \gamma$ and $W W Z^{0}$ couplings.

Although experiments at the CERN LEP-100 $e^{+} e^{-}$collider and the SLAC SLC $e^{+} e^{-}$ collider [11] have provided stringent tests [12,13] of the standard model of the electroweak interactions [14 it is mainly the fermion-gauge boson couplings that have been tested and the gauge sector of the standard model remains largely terra incognita. A stringent test 
of the gauge structure of the standard model is provided by the tri-linear gauge vertices (TGV's); the $\gamma W W$ and $Z W W$ vertices. Within the standard model, these couplings are uniquely determined by $S U(2)_{L} \times U(1)$ gauge invariance so that a precise measurement of the vertex poses a severe test of the gauge structure of the theory. If these couplings were observed to have different values than their standard model values, it would indicate the need for physics beyond the standard model.

A problem common to many processes used to study TGV's is that they involve both the $W W \gamma$ and $W W Z$ vertices making it difficult to disentangle the contributions. In this paper we study the sensitivity of the process $e^{+} e^{-} \rightarrow\left(Z, \gamma^{*}\right) \nu_{l} \bar{\nu}_{l} \rightarrow \mu^{+} \mu^{-} \nu_{l} \bar{\nu}_{l}$ to anomalous couplings in the $\gamma W W$ and $Z W W$ vertices. This process offers the possibility of studying the $Z W W$ vertex independently of the $\gamma W W$ vertex by imposing appropriate kinematic cuts to select the invariant mass of the $\mu^{+} \mu^{-}$pair. We start with $\sqrt{s}=200 \mathrm{GeV}$ appropriate to LEP200 since this machine will be operational in the relatively near future [15]. We then turn to the proposed JLC/NLC/CLIC $e^{+} e^{-}$colliders with possible center of mass energies of $\sqrt{s}=500 \mathrm{GeV}$ and $1 \mathrm{TeV}$ [1,16 18]. It is important to mention that we do not include any beamsstrahlung radiation effects in our calculation [19]. These effects are very much machine dependant (beam intensity, bunch geometry, etc ...) and known to be negligible at $200 \mathrm{GeV}$, and small at $500 \mathrm{GeV}$. However, although they can be quite important at $1000 \mathrm{GeV}$, there has been recent progress in strategies to minimize the effects of beamstrahlung radiation. Interestingly, such high energy colliders offer the possibility of studying the process $e \gamma \rightarrow W^{-} \nu_{e}$ or $\gamma \gamma \rightarrow W^{+} W^{-}$[8,20]. Both of these processes have been studied in detail and appear very promising.

The outline of this paper is as follows: In the next section we write down the effective Lagrangian we will be studying and the resulting Feynman rule to give our conventions. We also discuss the present constraints on TGV's and expected constraints from future experiments. In section III we examine in detail the process we are interested in; $e^{+} e^{-} \rightarrow$ $\mu^{+} \mu^{-} \nu \bar{\nu}$ and describe the method of calculation. In section IV we present our results for the three energy regimes that we investigated. We summarize our conclusions in section V. 


\section{THE $W W V$ EFFECTIVE VERTEX}

A particularly useful means of probing for physics at high energy scales is to use the language of effective Lagrangians [13,21]. An effective Lagrangian parametrizes in as modelindependent a way as possible the low-energy implications of new physics at a much higher scale, $M$. The effective Lagrangian offers a common language so the sensitivity of various experimental observables can be compared in a model-independent way.

There are several different effective Lagrangians in the literature used to describe the trilinear gauge boson vertices (TGV's). They differ in that they make different assumptions on the symmetries and particle content respected by the effective Lagrangian. In our analysis we use the most general parametrization possible that respects Lorentz invariance, electromagnetic gauge invariance, and CP invariance [22-24]. Because this general Lagrangian hides the $S U(2) \times U(1)$ symmetry observed at present energies and obscures the expected size of it's parameters, it has been the object of some criticism in the literature [25]. It is, in fact, equivalent to the alternative $S U(2) \times U(1)$ invariant nonlinearly realized Lagrangian written in the Unitary gauge upon suitable field redefinitions [26] and in general one can transform the parameters of one effective Lagrangian to the parameters of another [27]. We choose to use the general Lagrangian in our analysis since it has become the standard parametrization used in phenomenology and therefore makes the comparison of the sensitivity of different observables to the TGV's straightforward.

The most general $W W V$ vertex, satisfying Lorentz invariance, $\mathrm{U}(1)$ gauge invariance and $\mathrm{CP}$ conservation allows four free independent parameters, $\kappa_{\gamma}, \lambda_{\gamma}, \kappa_{Z}$ and $\lambda_{Z}$ when the $W$ bosons couple to essentially massless fermions which effectively results in $\partial_{\mu} W^{\mu}=$ 0 [22,23]. We do not consider $\mathrm{CP}$ violating operators in this paper as they are tightly constrained by measurement of the neutron electron dipole moment which constrains the two

CP violating parameters to $\left|\tilde{\kappa}_{\gamma}\right|,\left|\tilde{\lambda}_{\gamma}\right|<\mathcal{O}\left(10^{-4}\right)$ [28]. Therefore, the most general Lorentz and CP invariant vertex compatible with electromagnetic gauge invariance is commonly parametrized as 22,23]: 


$$
\mathcal{L}_{W W V}=-i g_{V}\left\{\left(W_{\mu \nu}^{\dagger} W^{\mu} V^{\nu}-W_{\mu}^{\dagger} V_{\nu} W^{\mu \nu}\right)+\kappa_{V} W_{\mu}^{\dagger} W_{\nu} F^{\mu \nu}-\frac{\lambda_{V}}{M_{W}^{2}} W_{\lambda \mu}^{\dagger} W_{\nu}^{\mu} F^{\nu \lambda}\right\}
$$

where $V$ represents either the photon or the $Z^{0}$ and $W^{\mu}$ the $W^{-}$fields. As usual, $W_{\mu \nu}=$ $\partial_{\mu} W_{\nu}-\partial_{\nu} W_{\mu}$ and $F_{\mu \nu}=\partial_{\mu} V_{\nu}-\partial_{\nu} V_{\mu}$ where $V$ is either the photon or the Z boson, $M_{W}$ is the $W$ boson mass, and $g_{\gamma}=e$ and $g_{Z^{0}}=e \cot \theta_{w}$. Higher dimension operators would correspond to momentum dependence in the form factors which we ignore. At tree level the standard model requires $\kappa_{V}=1$ and $\lambda_{V}=0$. Note that the presence of the $\mathrm{W}$-boson mass factor in the $\lambda_{V}$ term is ad hoc and one could argue that the scale $\Lambda$ of new physics would be more appropriate. We will conform to the usual parametrization and will not address this issue any further.

The resulting Feynman rule for the $W W V$ vertex is given below with the notation and conventions given in fig. 1.

$$
\begin{gathered}
i g_{V}\left\{g_{\alpha \beta}\left[\left(1-\tilde{\lambda} k_{-} \cdot q\right) k_{+\mu}-\left(1-\tilde{\lambda} k_{+} \cdot q\right) k_{-\mu}\right]\right. \\
-g_{\alpha \mu}\left[\left(1-\tilde{\lambda} k_{-} \cdot q\right) k_{+\beta}-\left(\kappa-\tilde{\lambda} k_{+} \cdot k_{-}\right) q_{\mu}\right]-g_{\beta \mu}\left[\left(\kappa-\tilde{\lambda} k_{-} \cdot k_{+}\right) q_{\alpha}-\left(1-\tilde{\lambda} k_{+} \cdot q\right) k_{-\alpha}\right] \\
\left.+\tilde{\lambda}\left(k_{+\mu} k_{-\alpha} q_{\beta}-k_{-\mu} q_{\alpha} k_{+\beta}\right)\right\}
\end{gathered}
$$

where $g_{V}=e$ for $V=\gamma$ and $e \cot \theta_{w}$ for $V=Z^{0}$ and $\tilde{\lambda}=\lambda / M_{W}^{2}$.

In the static limit (all particles on mass-shell), the parameters $\lambda_{\gamma}$ and $\kappa_{\gamma}$ are related to the anomalous magnetic and electric quadrupole moments of the $W$ boson by:

$$
\mu_{W}=\frac{e}{2 M_{W}}\left(1+\kappa_{\gamma}+\lambda_{\gamma}\right) \quad Q_{W}=\frac{-e}{2 M_{W}^{2}}\left(\kappa_{\gamma}-\lambda_{\gamma}\right)
$$

with similar expressions for the weak moments (i.e. those that involve the Z boson) At tree-level, the standard model requires $\kappa_{V}=1$ and $\lambda_{V}=0$. Higher order corrections to $\mu_{W}$ and $Q_{W}$ have been calculated in the past and the results are in the $2 \%$ range in the minimal standard model and in the $3 \%$ range in minimal supersymmetric extensions of the model [29].

Constraints can be obtained from precision measurements on the $W W \gamma$ and $W W Z^{0}$ vertices via loop corrections since deviations from their standard model values would have 
resulted in discrepancies of observables from their standard model predictions [30 32]. At present the limits on TGV's obtained from a global analysis of precision measurements are relatively weak; $\left|\delta \kappa_{\gamma}\right| \leq 0.12,\left|\delta \kappa_{Z}\right| \leq 0.08,\left|\lambda_{\gamma}\right| \leq 0.07$, and $\left|\lambda_{Z}\right| \leq 0.09$ at $95 \%$ C.L. varying one parameter at a time [30. In a simultaneous fit cancellations could lead to larger values and in addition, because there are ambiguities in the extraction of these bounds from loop calcuations due to ignorance of the operators values at high energy and the scale of new physics, the bounds obtained in this manner are at best order of magnitude estimates.

In contrast, direct measurements of gauge boson couplings are unambiguous. The only existing direct limits come from the measurement of associated $\gamma W$ production by the UA2 experiment at the CERN $\bar{p} p$ collider which obtained $-3.5<\kappa_{\gamma}<5.9$ and $-3.6<\lambda_{\gamma}<3.5$ at $95 \%$ C.L. [33. The limits obtained from the Tevatron are unsettled at present, with two theory analysis finding significantly different limits [34,35. The most optimistic limits from the Tevatron are $\left|\delta \kappa_{\gamma}\right| \simeq 3$ and $\left|\delta \lambda_{\gamma}\right| \simeq 1.2$ at $68 \%$ C.L. 35 The sensitivities expected at an upgraded Tevatron with $L=100 \mathrm{pb}^{-1}$ are $\left|\delta \kappa_{\gamma}\right| \simeq 1.4$ and $\left|\delta \lambda_{\gamma}\right| \simeq 0.47$ at $90 \%$ C.L. [35]. In the near future, HERA will be able to constrain the $\gamma W W$ vertex through single $\mathrm{W}$ production [36 38] and high $p_{T}$ photons [39]. Statistics will be the main limiting factor and a precision of \pm 0.5 or so is expected [36].

Putting tight constraints on the trilinear gauge boson couplings by studying $W$ pair production is one of primary motivations for the LEP200 upgrade 22, 15,40]. A precision of $30-40 \%$ is expected from a direct measurement of the cross-section. If one can reconstruct the W-bosons, their angular distribution offers a more sensitive probe and could lead to a bound of $25 \%$ or so. Another possibility it to study single $W$ production in $e-\gamma$ collisions [5.86,20]. The process $\gamma \gamma \rightarrow W^{+} W^{-}$through heavy ion collisions also offers interesting possibilities [41. However, one has to deal with an enormous background in the case of head-on collisions or a greatly reduced hard-photon rate for glancing collisions. In the longer term the LHC offers good possibilities. Baur and Zeppenfeld 42 have shown that a measurement of $\left|\delta \kappa_{\gamma}\right| \sim 0.2-0.5$ at $99.9 \% \mathrm{cl}$. or better is possible, assuming an integrated luminosity of $10^{4} \mathrm{pb}^{-1}$. 
In the far future there is growing interest in the physics that can be done at high energy $e^{+} e^{-}$colliders with $\sqrt{s}=500 \mathrm{GeV}$ or $\sqrt{s}=1 \mathrm{TeV}$, referred to as the Next Linear Collider (NLC), the Japan Linear Collider (JLC) or the CERN Linear Collider (CLIC) [1, 16 18]. Various options are being studied including $e \gamma$ collisions where the energetic photons are obtained either by backscattering a laser on one of the incident leptons or by beamstruhlung photons. Measurements at these colliders are very sensitive to anomalous couplings with $e \gamma$ collisions putting some of the more stringent bounds on anomalous $W W \gamma$ couplings [8,20].

\section{CALCULATIONS AND RESULTS}

The process $e^{+} e^{-} \rightarrow\left(\gamma^{*} Z\right) \nu_{l} \bar{\nu}_{l} \rightarrow \mu^{+} \mu^{-} \nu_{l} \bar{\nu}_{l}$ has several advantages; it is a t-channel process and does not decrease as quickly as s-channel processes as the c.m. energy increases. More importantly, it offers the possibility of isolating the $Z W W$ vertex from the $\gamma W W$ vertex by imposing appropriate cuts on the invariant mass of the $\mu^{+} \mu^{-}$pairs. One drawback is that a total of 28 Feynman diagrams contribute to the process and one has to add incoherently the three families of neutrinos. Although only the 2 diagrams shown in fig.2 (a) and 2(b) contribute to the vertex we wish to study, in order to properly take into account the non resonant backgrounds and maintain gauge invariance, at least in the standard model limit, we must include all 28 diagrams. We leave $\kappa_{V}$ and $\lambda_{V}$ as free parameters.

To evaluate the cross-sections and different distributions, we used the CALKUL helicity amplitude technique [45] to obtain expressions for the matrix elements and performed the

phase space integration using Monte Carlo techniques [46]. The expressions for the helicity amplitudes are lengthy and unilluminating so we do not include them here. The interested reader can obtain them directly from the authors. To obtain numerical results we used the values $\alpha=1 / 128, \sin ^{2} \theta=0.23, M_{Z}=91.187 \mathrm{GeV}, \Gamma_{Z}=2.5 \mathrm{GeV}, M_{W}=80.2 \mathrm{GeV}$, and $\Gamma_{W}=2.1 \mathrm{GeV}$.

The signal we are studying is an energetic $\mu^{+} \mu^{-}$pair plus missing transverse momentum due to the neutrinos coming from the original beams. In order to eliminate potential 
background from $e^{+} e^{-} \rightarrow e^{+} e^{-} \mu^{+} \mu^{-}$via two photons, where the $e^{+}$and $e^{-}$escape down the beam pipe, we require missing $\not p_{T} \geq 10 \mathrm{GeV}$. We also require $10 \leq E_{\mu^{ \pm}} \leq \sqrt{s} / 2-10$ $\mathrm{GeV}$, to avoid 2 body s-channel processes and box diagrams. Note that these kinematic cuts overlap. In order to take into account finite detector acceptance, we require that the $\mu^{+}$and $\mu^{-}$be at least 10 degrees away from the beam line. Our conclusions are not very sensitive to the exact values of these cuts.

In fig. 3, we show the cross-section for $e^{+} e^{-} \rightarrow \nu_{l} \bar{\nu}_{l} \mu^{+} \mu^{-}$as a function of $\sqrt{s}$ with the cuts described above and consider the effects of different cuts on the invariant mass of the $\mu^{+} \mu^{-}$pairs. We note that, as expected of typical t-channel behavior, the cross-section does not go down with energy as does the QED point cross-section. The cross-section for no cuts on $M_{\mu^{+} \mu^{-}}$(solid line) is considerable but it is dominated by low invariant mass events due to the photon pole which appears in many of the Feynman diagrams including photon bremsstrahlung and the diagram of interest in fig 2(a). For the most part the low invariant mass contributions are an unwanted background which obscures the physics we are interested in. Imposing a cut of $M_{\mu^{+} \mu^{-}}>25 \mathrm{GeV}$ eliminates this pole and reduces the cross-section substantially (long-dashed line). Finally, if we impose the cut that $M_{\mu^{+} \mu^{-}}$lies within $5 \mathrm{GeV}$ of the $\mathrm{Z}$ pole (dotted line) we can separate the effects of the ZWW vertex from the $\gamma W W$ vertex since the photon contribution is now smaller by a factor $\left(\Gamma_{Z} / M_{Z}\right)^{2}$. This last curve is in fact the superposition of two diagrams: the s-channel process of fig. (2b) which rises sharply above $200 \mathrm{GeV}$ and falls quickly at $\sqrt{s} \geq 300 \mathrm{GeV}$ and the W fusion diagrams that fall quickly below $200 \mathrm{GeV}$ and rise up to $1000 \mathrm{GeV}$. The cut $M_{\mu^{+} \mu^{-}} \geq 25$ $\mathrm{GeV}$ is an intermediate state between this extreme and the photon-dominated case where the photon bremstrahlung diagrams dominate for the entire energy range.

It is clear from this figure that this process is hopeless for LEP-100; without any cut on $M_{\mu^{+} \mu^{-}}$the cross-section is substantial, but rather insensitive to variations on $\kappa_{V}$ and $\lambda_{V}$. Imposing a cut on the invariant mass increases the sensitivity to anomalous couplings but reduces the cross-section to an unmeasurable level. 


\section{A. $\sqrt{s}=200 \mathrm{GeV}$}

For $\sqrt{s}=200 \mathrm{GeV}$ we use the kinematic cuts $5<E_{\mu^{ \pm}}<95 \mathrm{GeV}$ and $p_{T}>5 \mathrm{GeV}$. The cross section with these cuts and in addition, cuts on $M_{\mu^{+} \mu^{-}}$of no cut, $M_{\mu^{+} \mu^{-}}>10 \mathrm{GeV}$, and $86 \mathrm{GeV}<M_{\mu^{+} \mu^{-}}<96 \mathrm{GeV}$ are $1.9 \mathrm{pb}, 0.23 \mathrm{pb}$, and $0.035 \mathrm{pb}$ respectively. The latter cut would isolate the effects of the $W W Z$ vertex from that of the $W W \gamma$ vertex. Unfortunately, for the parameters of LEP-200 $\left(\sqrt{s}=200 \mathrm{GeV}\right.$, and an integrated luminosity of $\left.250 p b^{-1}\right)$ the number of events remaining after these cuts is not statistically useful.

To maximize the sensitivity to anomalous TGV couplings we examined numerous kinematic distributions. The two which best separated the uninteresting photon bremstrahlung contribution from signals for anomalous couplings are $d \sigma / d M_{\mu^{+} \mu^{-}}$and $d \sigma / d \cos \theta_{\mu^{+} \mu^{-}}$which are shown in fig. 4 for several values of $\kappa_{\gamma}, \lambda_{\gamma}, \kappa_{Z}$ an $\lambda_{Z}$. It is clear from fig. (4a) that sensitivity to anomalous couplings is due to interference between the photon and $Z^{0}$ propagators. We therefore examined the effects of removing the contribution of the $Z^{0}$ pole; $10<M_{\mu^{+} \mu^{-}}<88 \mathrm{GeV}$. Although this increased the "signal to background" it also reduced the cross section. Once realistic efficiencies are considered we do not feel that enough events would be left to improve the measurement of the TGV's. We included the cuts of $M_{\mu^{+} \mu^{-}}>10 \mathrm{GeV}$ and $\cos \theta_{\mu^{+} \mu^{-}}<0.95$ in our subsequent calculations used to determine the sensitivity of the measurements to anomalous couplings. We note that these two cuts correspond to the same region in phase space and therefore overlap. In addition, deviations from the standard model show up in various distributions such as $d \sigma / d \cos \theta_{\mu^{+} \mu^{-}}$and we could bin these distributions to perform a $\chi^{2}$ analysis. In practice, however, the cross section is too small at $\sqrt{s}=200 \mathrm{GeV}$ to improve the sensitivity.

Since there are four free parameters, the parameter space is four dimensional which can be projected onto six 2-dimensional planes. We performed an extensive search in the parameter space and found that to a good approximation the largest ellipse in any given plane is reached when the other two parameters are kept at their standard model values. The small exception is the relative insensitivity to variations in $\kappa_{\gamma}$ which results in a very small 
enlargement of the boundary for non-standard model values along the $\kappa_{\gamma}$ axis. The $95 \%$ C.L. for integrated luminosities of $250 \mathrm{pb}^{-1}$ and $500 \mathrm{pb}^{-1}$ for the two planes, $\kappa_{Z}$ versus $\lambda_{Z}$ and $\kappa_{\gamma}$ versus $\lambda_{\gamma}$ are shown in fig. 5. These bounds represent the regions of the parameter space that can be ruled out as inconsistent with the standard model for a measurement of the standard model values for the given integrated luminosity. If we vary one parameter at a time and hold the rest at their standard model values we obtain the limits, based on the statistical error obtained from an integrated luminosity of $500 \mathrm{pb}^{-1}, \delta \kappa_{\gamma}={ }_{-1.0}^{+1.9}, \delta \lambda_{\gamma}={ }_{-1.3}^{+0.9}$, $\delta \kappa_{Z}= \pm 1.0, \delta \lambda_{Z}= \pm 0.8$ at $95 \%$ C.L.. In this analysis we did not assume any constraints on the parameters. Imposing a custodial $S U(2)$ symmetry gives the relation $\lambda_{Z} \simeq \lambda_{\gamma}$ [47. If we also take $\kappa_{Z}=\kappa_{\gamma}$ we obtain the sensitivities $\delta \lambda={ }_{-0.7}^{+0.6}$ and $\delta \kappa={ }_{-0.6}^{+1.0}$ at $95 \%$ C.L. which is not so different from the unconstrained result for the $Z$ parameters but significantly tighter than the unconstrained results we obtained for $\kappa_{\gamma}$ and $\lambda_{\gamma}$. Since the contour axes are almost aligned with the parameter axes two planes contain most, if not all the information about the limits on the 4 parameters.

At $200 \mathrm{GeV}$ we find that the constraints that can be achieved using the process $e^{+} e^{-} \rightarrow$ $\mu^{+} \mu^{-} \nu \bar{\nu}$ cannot compete with the constraints obtained from $\mathrm{W}$-pair production. Therefore, at best this process would provide a consistency check for other measurements.

\section{B. $\sqrt{s}=500 \mathrm{GeV}$}

We next turn to an "NLC" type $e^{+} e^{-}$collider with $\sqrt{s}=500 \mathrm{GeV}$. We consider integrated luminosities of 10 and $50 \mathrm{fb}^{-1}$ and use the kinematic cuts $10 \mathrm{GeV}<E_{\mu^{ \pm}}<240 \mathrm{GeV}$ and $\not p_{T}>10 \mathrm{GeV}$. With these cuts we obtain the invariant mass distribution, $d \sigma / d M_{\mu^{+} \mu^{-}}$shown in fig. 6 .

The increased cross section and expected high luminosity at the NLC leads to a significantly larger number of events making it possible to study the reaction at the $Z^{0}$ pole, significantly reducing the contributions of the $\gamma W W$ vertex to the process. Although results have been presented previously for $\left|M_{\mu^{+} \mu^{-}}-M_{Z}\right|<5 \mathrm{GeV}$ [7] the lumininosity that the 
NLC is expected to achieve is significantly higher than what was used in the earlier analysis. We have therefore revised the analysis taking into account the higher luminosity and emphasizing its implications before proceeding to the results off the $Z^{0}$ resonance. In particular we will see that systematic errors will play an increasingly important role in precision measurments.

For the cut $\left|M_{\mu^{+} \mu^{-}}-M_{Z}\right|<5 \mathrm{GeV}$ we verified that the cross section and distributions are insensitive to variations in $\kappa_{\gamma}$ and $\lambda_{\gamma}$. We considered the effects of varying $\kappa_{Z}$ and $\lambda_{Z}$ on the cross section $\sigma\left(e^{+} e^{-} \rightarrow \mu^{+} \mu^{-} \nu_{l} \bar{\nu}_{l}\right)$ and found that varying one parameter at a time we obtained a sensitivity (in the sense of consistency with the standard model) of $\delta \kappa_{Z}= \pm 0.1$ $\left(\lambda_{Z}=0\right)$ and $\delta \lambda_{Z}={ }_{-0.5}^{+0.2}\left(\kappa_{Z}=1\right)$ at $95 \%$ C.L. based on $20 \mathrm{fb}^{-1}$ integrated luminosity. However, when we let both parameters vary at the same time we find that regions in the parameter space very far from the standard model give cross sections consistent with the standard model value [7]. To eliminate the ambiguities we examined a number of kinematic distributions. The most sensitive are the angular distribution of the muons with respect to each other $\left(\theta_{\mu^{+} \mu^{-}}\right)$and the transverse momentum of the reconstructed $Z^{0}$ boson $\left(p_{T Z}\right)$ which we show in fig. 7 for several values of $\kappa_{Z}$ and $\lambda_{Z}$. We performed a $\chi^{2}$ analysis based on the angular distribution using the bins; $-1.0<\theta_{\mu^{+} \mu^{-}}<-0.5,-0.5<\theta_{\mu^{+} \mu^{-}}-0.1$, and $-0.1<\theta_{\mu^{+} \mu^{-}}<0.75$ and another one based on the $p_{T Z}$ distribution with the bins; $p_{T Z}<80$ $\mathrm{GeV}, 80 \mathrm{GeV}<p_{T Z}<120 \mathrm{GeV}$, and $120 \mathrm{GeV}<p_{T Z}<240 \mathrm{GeV}$. The $68 \%, 90 \%$, and $95 \%$ C.L. bounds using $d \sigma / d \cos \theta_{\mu^{+} \mu^{-}}$and $d \sigma / d p_{T Z}$ based on $10 \mathrm{fb}^{-1}$ are shown in fig. (8a) and (8b) respectively. This additional information substantially restricts the allowed region in parameter space that is consistent with the standard model with $\delta \kappa_{Z}= \pm 0.2$ and $\delta \lambda={ }_{-0.4}^{+0.3}$ at $95 \%$ C.L. .

These results were based on $10 \mathrm{fb}^{-1}$ of integrated luminosity. It is expected that the luminosity is likely to be higher than this which would improve the measurement capabilities. On the other hand we have neglected systematic errors in our analysis. Monte Carlo studies of SLD type detectors give very crude estimates of systematic errors of $5 \%$ for cross section measurements 48]. In fig. 9 we show the $95 \%$ C.L. assuming $10 \mathrm{fb}^{-1}$ and $50 \mathrm{fb}^{-1}$ with and 
without a $5 \%$ systematic measurement error. We find that although improving the statistical error improves the sensitivity the systematic error tends to be more important. In other words, one gains more by reducing the systematic error than by significantly increasing the luminosity.

Although these results are no improvement over the expected LEP200 measurements based on $W^{+} W^{-}$pair production, they offer a means of measuring the $W W Z$ vertex independently of the $W W \gamma$ vertex.

Whereas isolating the $Z^{0}$ pole offers a means of studying the $W W Z$ vertex independently of the $W W \gamma$ vertex, there is a severe penalty in terms of reduced cross section and sensitivity to anomalous coupling. We therefore examine less restrictive cuts on the $\mu^{+} \mu^{-}$invariant mass which restores the $W W \gamma$ vertex. We searched for the range of $M_{\mu^{+} \mu^{-}}$which exhibited the largest sensitivity to anomalous couplings and found it to be $110 \leq M_{\mu^{+}} \mu^{-} \leq 400 \mathrm{GeV}$. As before, with four independant parameters, an extensive search in the parameter space showed that, to a good approximation, the largest (weakest) confidence limit bounds in any two parameters are reached when the other two parameters are kept at their standard model values. The contours for $\kappa_{\gamma}$ vs $\lambda_{\gamma}$ and $\kappa_{Z}$ vs $\lambda_{Z}$ are shown in fig. (10a) and (10b) respectively, based on the statistics from $10 \mathrm{fb}^{-1}$ integrated luminosity. These bounds were improved slightly when we relaxed the acceptance cuts around the beam axis to $|\cos \theta|<0.8$ from $\cos \theta>10^{\circ}$. We examined other kinematic distributions but found that for the parameter range allowed by fig. 10, the distributions are very similar and did not offer a significant improvement of the bounds obtained from the cross section measurement. (Imposing the kinematic cuts $E_{Z}<300 \mathrm{GeV}$ gave a very slight improvement and $p_{T Z}>100 \mathrm{GeV}$ distorted the ellipses to give a slight improvement on $\delta \lambda_{Z}$ )

Finally, we binned the $\mu^{+} \mu^{-}$invariant mass distribution into the four bins; $25<M_{\mu^{+} \mu^{-}}<$ 86, $86<M_{\mu^{+} \mu^{-}}<96,96<M_{\mu^{+} \mu^{-}}<110$, and $110<M_{\mu^{+} \mu^{-}}<400$ and performed a $\chi^{2}$ analysis, varying one parameter at a time. The resulting 95\% C.L. bounds based on $\mathrm{L}=10 \mathrm{fb}^{-1}$ and $\mathrm{L}=50 \mathrm{fb}^{-1}$ with and without systematic errors are summarized in Table I along with our other results for $\sqrt{s}=500 \mathrm{GeV}$. As before, one should be cautious about 
possible ambiguities when intepreting the results obtained by varying only one parameter at a time. Only the results for $\mathrm{L}=50 \mathrm{fb}^{-1}$ approach the sensitivity required to observe loop contributions to the TGV's. However, when systematic errors are included it is unlikely that these measurements will reveal non-standard model physics through radiative corrections to the TGV's. In addition the systematic errors are the limiting factor in the sensitivities, not the statistical errors.

$$
\text { C. } \sqrt{s}=1 \mathrm{TeV}
$$

The final case we consider is a $1 \mathrm{TeV} e^{+} e^{-}$collider. Although beamsstrahlung effects are known to be important in this energy regime [19], we will neglect them since they depend on many machine dependent factors making it difficult to estimate at this time. In any case, much progress has been made in understanding how to eliminate beamstrahlung so it may not be as important a factor as originally feared. This should be kept in mind when assessing our results. In what follows we use the kinematic cuts of $p_{T}$ of $10 \mathrm{GeV}, \theta_{\mu^{ \pm}}>10^{\circ}$, and $10<E_{\mu^{ \pm}}<490 \mathrm{GeV}$.

As before, we imposed a cut on $M_{\mu^{+} \mu^{-}}$to isolate the $Z^{0} W W$ vertex. The standard model cross-section is $0.52 \mathrm{pb}$. We verified explicitly that varying $\kappa_{\gamma}$ and $\lambda_{\gamma}$ by $10 \%$ changed the total cross-section by 2 part in 10,000 or less as expected. Varying $\kappa_{Z}$ and $\lambda_{Z}$ by $10 \%$ changed the total cross-section by $2 \%$ or less. This small variation does not translate into particularly significant bounds on the TGV's. As before we performed a $\chi^{2}$ analysis based on four equal bins for $\theta_{\mu^{+} \mu^{-}}$and the four bins $p_{T Z}<125 \mathrm{GeV}, 125 \mathrm{GeV}<p_{T Z}<250 \mathrm{GeV}, 250$ $\mathrm{GeV}<p_{T Z}<375 \mathrm{GeV}$, and $p_{T Z}>375 \mathrm{GeV}$. The $68 \%, 90 \%$, and $95 \%$ C.L. bounds using $d \sigma / d \cos \theta_{\mu^{+} \mu^{-}}$and $d \sigma / d p_{T Z}$ based on $50 \mathrm{fb}^{-1}$ are shown in fig. (11a) and (11b) respectively. Although the TGV's can be constrained to less than about 0.07 with $50 \mathrm{fb}^{-1}$ and less than about 0.04 with $200 \mathrm{fb}^{-1}$, when a $5 \%$ systematic error is included these bounds weaken to about 0.12 .

Given these relatively weak bounds we concentrated on large invariant mass where there can be interference between the two vertices so that although the cross-section is reduced, 
the sensitivity to anomalous coupling is greatly increased. As seen on fig. 12, the greatest sensitivity to anomalous coupling occurs in the mass range $200 \leq M_{\mu^{+}} \mu^{-} \leq 900 \mathrm{GeV}$ although the upper bound could be pushed to $1 \mathrm{TeV}$ without changing our results. Note that the values used here $(\delta \kappa=\delta \lambda= \pm 0.2)$ are extreme and are used simply to illustrate our point. The resulting standard model cross-section is $0.024 \mathrm{pb}$. We show on fig. 13 two of the six planes of the four dimensional parameter space. Over this small range in parameter space, the shapes of the different distributions are very similar and one would not gain much (if anything at all) by considering them over a total cross-section measurement. The 95\% C.L. bounds that we obtain assuming $50 \mathrm{fb}^{-1}$ and no systematic errors, varying one parameter at a time are: $\delta \kappa_{\gamma}={ }_{-0.01}^{+0.04}, \delta \kappa_{Z}= \pm 0.01, \delta \lambda_{\gamma}={ }_{-0.01}^{+0.03}$, and $\delta \lambda_{Z}{ }_{-0.01}^{+0.02}$. For comparison, the bounds obtained by assuming $\kappa_{Z}=\kappa_{\gamma}$ and $\lambda_{Z}=\lambda_{\gamma}$ imposed by custodial SU(2) symmetry are slightly stronger with $\delta \kappa={ }_{-0.006}^{+0.03}$ and $\delta \lambda={ }_{-0.006}^{+0.008}$. Again, we emphasize that one must be cautious in interpreting bounds obtained by varying one parameter at a time. The bounds that can be obtained with the various kinematic cuts, luminosities, with and without systematic errors are summarized in Table II. It is clear that the greatly improved sensitivity to anomalous coupling more than compensates for the reduced cross-section.

\section{CONCLUSIONS}

We have shown that the process $e^{+} e^{-} \rightarrow \mu^{+} \mu^{-} \nu \bar{\nu}$ can be useful in disentangling the different contributions to the $\gamma W^{+} W^{-}$and $Z^{0} W^{+} W^{-}$vertices. We included all processes that lead to this four-fermion final state. Appropriate cuts on the invariant mass of the muon pairs offer the possibility of measuring the $Z^{0} W^{+} W^{-}$vertex by itself albeit with reduced precision. This is due to both reduced statistics but also due to reduced sensitivity of the process to anomalous couplings. With the cut $\left|M_{\mu^{+} \mu^{-}}-M_{Z}\right|<5 \mathrm{GeV}$ the cross section is dominated by the one diagram and gauge vertex we are interested in so that there are

\footnotetext{
${ }^{3}$ Strictly speaking custodial SU(2) imposes $\lambda_{Z}=\lambda_{\gamma}$ and $\kappa_{Z}=\kappa_{\gamma}=1$ [47.
} 
no sensitive gauge cancellations in the process. On the other hand, off the $Z^{0}$ resonance the anomalous couplings can interfere with other diagrams resulting in greater sensitivity to anomalous couplings.

The process turned out to be hopeless at LEP-100 because of the low cross section. At LEP-200 it can lead to, at best, a consistency check of bounds extracted from $W$-pair production. At higher energy $e^{+} e^{-}$colliders, it can lead to very stringent bounds precise enough to test the TGV's at the level of radiative corrections. The bounds were obtained using many different measurements such as the angular distributions of the outgoing muons, the transverse momentum distribution of the reconstructed $Z^{0}$ boson, and the integrated cross section for the process off the $Z^{0}$ resonance.

Studying the four-fermion final state and including all diagrams which contributes to the final state leads to a much richer phenomenology than would be obtained by simply studying final state gauge bosons. Thus, the entire process, with all the contributing diagrams, should be studied when examining the physics potential of a specific reaction. We used the high luminosities planned for at the high energy $e^{+} e^{-}$colliders to estimate statistical errors. However, when we included reasonable estimates of systematic errors we found that the limiting factor in high precision measurements will likely be systematic errors not statistical errors. The challenge will therefore be to reduce the systematic errors and one should be very careful with respect to the conclusions one makes by only considering statistical errors.

\section{ACKNOWLEDGMENTS}

This research was supported in part by the Natural Sciences and Engineering Research Council of Canada and Les Fonds FCAR du Quebec. The authors gratefully acknowledge the contributions of Randy Lewis in the early stages of this work. G.C. wants to thank the Superconducting Supercollider Laboratory for the use of their computing facilities. S.G. thanks Tim Barklow and Dean Karlen for helpful conversations and Drew Peterson for assistance in preparing the figures. 


\section{The Helicity Amplitudes}

In this appendixt, we outline the use of the CALKUL spinor technique. We limit our discussion to massless fermions which apply to our problem. The propagators for the fermions and gauge bosons have the same form as in the trace technique so we do not discuss them here.

The spinor technique results in reducing strings of spinors and gamma matrices to sandwiches of spinors which can be evaluated easily. In doing so, one makes extensive use of the left and right projection operators defined by $\omega_{ \pm}=\frac{1}{2}\left(1 \pm \gamma_{5}\right)$. One defines two four-vectors, $k_{0}^{\mu}$ and $k_{1}^{\mu}$, which obey the following relations:

$$
k_{0} \cdot k_{0}=0, \quad k_{1} \cdot k_{1}=-1, \quad k_{0} \cdot k_{1}=0
$$

and the basic spinors as:

$$
u_{-}\left(k_{0}\right) \bar{u}_{-}\left(k_{0}\right)=\omega_{-} \not k_{0}
$$

and

$$
u_{+}\left(k_{0}\right)=\not k_{1} u_{-}\left(k_{0}\right)
$$

Note that in the massless limit, one can use $u$ and $\bar{u}$ to describe both particles and antiparticles, with the spin sum $\sum_{\lambda} u_{\lambda}(p) \bar{u}_{\lambda}(p)=\not p$. These two spinors are the building blocks for any spinor of lightlike momentum $\mathrm{p}$ :

$$
u_{\lambda}(p)=\frac{\not p u_{-\lambda}\left(k_{0}\right)}{\sqrt{2 p \cdot k_{0}}}
$$

Two identities are essential for the reduction of the strings; the spin sum given above and the Chisholm identity:

$$
\bar{u}_{\lambda}\left(p_{1}\right) \gamma^{\mu} u_{\lambda}\left(p_{2}\right) \gamma_{\mu} \equiv 2 u_{\lambda}\left(p_{2}\right) \bar{u}_{\lambda}\left(p_{1}\right)+2 u_{-\lambda}\left(p_{1}\right) \bar{u}_{-\lambda}\left(p_{2}\right)
$$

where $\lambda$ is \pm 1 and represents the helicity state. These two identities allow one to reduce strings of spinors and gamma matrices to sandwiches of spinors. Only two of the four

\footnotetext{
${ }^{4}$ Note: This appendix does not appear in the version submitted to the journal.
} 
possible sandwiches are non-zero:

$$
s\left(p_{1}, p_{2}\right) \equiv \bar{u}_{+}\left(p_{1}\right) u_{-}\left(p_{2}\right)=-s\left(p_{2}, p_{1}\right)
$$

and

$$
t\left(p_{1}, p_{2}\right) \equiv \bar{u}_{-}\left(p_{1}\right) u_{+}\left(p_{2}\right)=s\left(p_{2}, p_{1}\right)^{*} .
$$

Once the amplitude has been reduced to a series of factors of $s\left(p_{i}, p_{j}\right)$ and $t\left(p_{k}, p_{l}\right)$, the expressions can be evaluated by computer. A judicious choice of the four-vectors $k_{0}^{\mu}$ and $k_{1}^{\mu}$ simplifies the evaluation of the $s$ and $t$ terms. For our calculation, we use;

$$
\begin{gathered}
p_{i}^{\mu}=\left(p_{i}^{0}, p_{i}^{x}, p_{i}^{y}, p_{i}^{z}\right) \\
k_{0}^{\mu}=(1,1,0,0) \\
k_{1}^{\mu}=(0,0,1,0)
\end{gathered}
$$

to obtain

$$
s\left(p_{1}, p_{2}\right)=\left(p_{1}^{y}+i p_{1}^{z}\right) \frac{\sqrt{p_{2}^{0}-p_{2}^{x}}}{\sqrt{p_{1}^{0}-p_{1}^{x}}}-\left(p_{2}^{y}+i p_{2}^{z}\right) \frac{\sqrt{p_{1}^{0}-p_{1}^{x}}}{\sqrt{p_{2}^{0}-p_{2}^{x}}}
$$

These forms are ideally suited for programming. When dealing with several diagrams, one simply evaluates the amplitudes of each diagram as complex numbers and squares the sum of the amplitudes in order to obtain the $\mid$ amplitude $\left.\right|^{2}$.

Using this technique and the following definitions

$$
e^{-}\left(p_{1}\right)+e^{+}\left(p_{2}\right) \rightarrow \mu^{-}\left(p_{3}\right)+\mu^{+}\left(p_{4}\right)+\nu_{l}\left(p_{5}\right)+\bar{\nu}_{l}\left(p_{6}\right)
$$

we obtain for the helicity amplitudes,

$$
\begin{aligned}
& M_{L L}^{a}=-2 i g^{2} g_{V}^{2} C_{L}^{V e} D_{W}\left(p_{1}-p_{5}\right) D_{W}\left(p_{2}-p_{6}\right) D_{V}\left(p_{3}+p_{4}\right) \\
&\left\{t ( 5 , 2 ) s ( 6 , 1 ) * \left[\left(1.0+\tilde{\lambda}_{V} p_{W^{+}} \cdot p_{Z}\right) *(t(3,1) s(1,4)-t(3,5) s(5,4))\right.\right. \\
&\left.-\left(1.0+\tilde{\lambda}_{V} p_{W^{-}} \cdot p_{Z}\right) *(t(3,2) s(2,4)-t(3,6) s(6,4))\right] \\
&+t(5,3) s(4,1) *\left[\left(\kappa_{V}+\tilde{\lambda}_{V} p_{W^{-}} \cdot p_{W^{+}}\right) *(-t(2,4) s(4,6)-t(2,3) s(3,6))\right. \\
&\left.-\left(1.0+\tilde{\lambda} p_{W^{+}} \cdot p_{Z}\right) *(t(2,1) s(1,6)-t(2,5) s(5,6))\right]
\end{aligned}
$$




$$
\begin{aligned}
& +t(2,3) s(4,6) *\left[\left(1.0+\tilde{\lambda}_{V} p_{W^{-}} \cdot p_{Z}\right) *(t(5,2) s(2,1)-t(5,6) s(6,1))\right. \\
& \left.-\left(\kappa_{V}+\tilde{\lambda} p_{W^{+}} \cdot p_{W^{-}}\right) *(-t(5,4) s(4,1)-t(5,3) s(3,1))\right] \\
& -\frac{1}{2} \tilde{\lambda}_{V} *[t(5,2) s(2,1)-t(5,6) s(6,1)] *[-t(2,4) s(4,6)-t(2,3) s(3,6)] \\
& *[t(3,1) s(1,4)-t(3,5) s(5,4)] \\
& +\frac{1}{2} \tilde{\lambda}_{V} *[-t(5,4) s(4,1)-t(5,3) s(3,1)] *[t(2,1) s(1,6)-t(2,5) s(5,6)] \\
& *[t(3,2) s(2,4)-t(3,6) s(6,4)]\} \\
& M_{L R}^{a}=-2 i g^{2} g_{V}^{2} C_{R}^{V e} D_{W}\left(p_{1}-p_{5}\right) D_{W}\left(p_{2}-p_{6}\right) D_{V}\left(p_{3}+p_{4}\right) \\
& \left\{t ( 5 , 2 ) s ( 6 , 1 ) \left[\left(1.0+\tilde{\lambda}_{V} p_{W^{+}} \cdot p_{Z}\right) *(s(3,1) t(1,4)-s(3,5) t(5,4))\right.\right. \\
& \left.-\left(1.0+\tilde{\lambda}_{V} p_{W^{-}} \cdot p_{Z}\right) *(s(3,2) t(2,4)-s(3,6) t(6,4))\right] \\
& +t(5,4) s(3,1) *\left[\left(\kappa_{V}+\tilde{\lambda}_{V} p_{W^{-}} \cdot p_{W^{+}}\right) *(-t(2,4) s(4,6)-t(2,3) s(3,6))\right. \\
& \left.-\left(1.0+\tilde{\lambda}_{V} p_{W^{+}} \cdot p_{Z}\right) *(t(2,1) s(1,6)-t(2,5) s(5,6))\right] \\
& +t(2,4) s(3,6) *\left[\left(1.0+\tilde{\lambda}_{V} p_{W^{-}} \cdot p_{Z}\right) *(t(5,2) s(2,1)-t(5,6) s(6,1))\right. \\
& \left.+\left(\kappa_{V}+\tilde{\lambda}_{V} p_{W^{-}} \cdot p_{W+}\right) *(t(5,4) s(4,1)+t(5,3) s(3,1))\right] \\
& -\frac{1}{2} \tilde{\lambda}_{V} *[t(5,2) s(2,1)-t(5,6) s(6,1)] *[-t(2,4) s(4,6)-t(2,3) s(3,6)] \\
& *[s(3,1) t(1,4)-s(3,5) t(5,4)] \\
& +\frac{1}{2} \tilde{\lambda}_{V} *[-t(5,4) s(4,1)-t(5,3) s(3,1)] *[t(2,1) s(1,6)-t(2,5) s(5,6)] \\
& *[s(3,2) t(2,4)-s(3,6) t(6,4)]\} \\
& M_{L L}^{b}=-i g^{2} g_{V}^{2} C_{L}^{V e} D_{W}\left(p_{3}+p_{6}\right) D_{W}\left(p_{4}+p_{5}\right) D_{V}\left(p_{1}+p_{2}\right) \\
& \left\{t ( 3 , 5 ) s ( 4 , 6 ) * \left[\left(1.0+\tilde{\lambda}_{V} p_{W^{+}} \cdot p_{Z}\right) *(-t(2,3) s(3,1)-t(2,6) s(6,1))\right.\right. \\
& \left.-\left(1.0+\tilde{\lambda}_{V} p_{W^{-}} \cdot p_{Z}\right) *(-t(2,4) s(4,1)-t(2,5) s(5,1))\right] \\
& +t(2,3) s(6,1) *\left[\left(\kappa_{V}+\tilde{\lambda}_{V} p_{W^{-}} \cdot p_{W+}\right) *(t(5,2) s(2,4)+t(5,1) s(1,4))\right. \\
& \left.-\left(1.0+\tilde{\lambda}_{V} p_{W^{+}} \cdot p_{Z}\right) *(-t(5,3) s(3,4)-t(5,6) s(6,4))\right] \\
& +t(5,2) s(1,4) *\left[\left(1.0+\tilde{\lambda}_{V} p_{W^{-}} \cdot p_{Z}\right) *(-t(3,4) s(4,6)-t(3,5) s(5,6))\right. \\
& \left.-\left(\kappa_{V}+\tilde{\lambda}_{V} p_{W^{-}} \cdot p_{W+}\right) *(t(3,2) s(2,6)+t(3,1) s(1,6))\right]
\end{aligned}
$$




$$
\begin{aligned}
& -\frac{1}{2} \tilde{\lambda}_{V} *[t(2,3) s(3,1)+t(2,6) s(6,1)] *[t(3,4) s(4,6)+t(3,5) s(5,6)] \\
& *[t(5,2) s(2,4)+t(5,1) s(1,4)] \\
& +\frac{1}{2} \tilde{\lambda}_{V} *[t(2,4) s(4,1)+t(2,5) s(5,1)] *[t(3,2) s(2,6)+t(3,1) s(1,6)] \\
& *[t(5,3) s(3,4)+t(5,6) s(6,4)]\} \\
& M_{R L}^{b}=-i g^{2} g_{V}^{2} C_{R}^{V e} D_{W}\left(p_{3}+p_{6}\right) D_{W}\left(p_{4}+p_{5}\right) D_{V}\left(p_{1}+p_{2}\right) \\
& \left\{t ( 3 , 5 ) s ( 4 , 6 ) * \left[\left(1.0+\tilde{\lambda}_{V} p_{W^{+}} \cdot p_{Z}\right) *(-s(2,3) t(3,1)-s(2,6) t(6,1))\right.\right. \\
& \left.-\left(1.0+\tilde{\lambda}_{V} p_{W^{-}} \cdot p_{Z}\right) *(-s(2,4) t(4,1)-s(2,5) t(5,1))\right] \\
& +t(3,1) s(2,6) *\left[\left(\kappa_{V}+\tilde{\lambda}_{V} p_{W^{-}} \cdot p_{W+}\right) *(t(5,2) s(2,4)+t(5,1) s(1,4))\right. \\
& \left.-\left(1.0+\tilde{\lambda}_{V} p_{W^{+}} \cdot p_{Z}\right) *(-t(5,3) s(3,4)-t(5,6) s(6,4))\right] \\
& +t(5,1) s(2,4) *\left[\left(1.0+\tilde{\lambda}_{V} p_{W^{-}} \cdot p_{Z}\right) *(-t(3,4) s(4,6)-t(3,5) s(5,6))\right. \\
& \left.-\left(\kappa_{V}+\tilde{\lambda}_{V} p_{W^{-}} \cdot p_{W+}\right) *(t(3,2) s(2,6)+t(3,1) s(1,6))\right] \\
& -\frac{1}{2} \tilde{\lambda}_{V} *[s(2,3) t(3,1)+s(2,6) t(6,1)] *[t(3,4) s(4,6)+t(3,5) s(5,6)] \\
& *[t(5,2) s(2,4)+t(5,1) s(1,4)] \\
& +\frac{1}{2} \tilde{\lambda}_{V}[s(2,4) t(4,1)+s(2,5) t(5,1)] *[t(3,2) s(2,6)+t(3,1) s(1,6)] * \\
& {[(t(5,3) s(3,4)+t(5,6) s(6,4)]\}} \\
& M_{L L}^{c}=2 i g^{2} g_{V}^{2} C_{L}^{V e} C_{L}^{V e} D_{f}\left(p_{2}-p_{4}-p_{3}\right) D_{V}\left(p_{3}+p_{4}\right) D_{W}\left(p_{1}-p_{5}\right) \\
& t(2,3) s(1,6) *[s(4,2) t(2,5)-s(4,3) t(3,5)] \\
& M_{L R}^{c}=2 i g^{2} g_{V}^{2} C_{L}^{V e} C_{R}^{V e} D_{f}\left(p_{2}-p_{3}-p_{4}\right) D_{V}\left(p_{3}+p_{4}\right) D_{W}\left(p_{1}-p_{5}\right) \\
& t(2,4) s(1,6) *[s(3,2) t(2,5)-s(3,4) t(4,5)] \\
& M_{L L}^{c-2}=2 i g^{2} g_{Z}^{2} C_{L}^{Z \nu} C_{L}^{Z e} D_{f}\left(p_{6}+p_{3}+p_{4}\right) D_{Z^{0}}\left(p_{3}+p_{4}\right) D_{W}\left(p_{1}-p_{5}\right) \\
& t(2,5) s(4,6) *[s(1,6) t(6,3)+s(1,4) t(4,3)] \\
& M_{L R}^{c-2}=2 i g^{2} g_{Z}^{2} C_{L}^{Z \nu} C_{R}^{Z e} D_{f}\left(p_{6}+p_{3}+p_{4}\right) D_{Z^{0}}\left(p_{3}+p_{4}\right) D_{W}\left(p_{1}-p_{5}\right) \\
& t(2,5) s(3,6) *[s(1,6) t(6,4)+s(1,3) t(3,4)] \\
& M_{L L}^{c-3}=-2 i g^{2} g_{Z}^{2} C_{L}^{Z \nu} C_{L}^{Z e} D_{f}\left(p_{5}+p_{4}+p_{3}\right) D_{Z^{0}}\left(p_{3}+p_{4}\right) D_{W}\left(p_{2}-p_{6}\right)
\end{aligned}
$$




$$
\begin{aligned}
& t(5,3) s(6,1) *[s(4,5) t(5,2)+s(4,3) t(3,2)] \\
& M_{L R}^{c-3}=-2 i g^{2} g_{Z}^{2} C_{L}^{Z \nu} C_{R}^{Z e} D_{f}\left(p_{5}+p_{4}+p_{3}\right) D_{Z^{0}}\left(p_{3}+p_{4}\right) D_{W}\left(p_{2}-p_{6}\right) \\
& t(5,4) s(6,1) *[s(3,5) t(5,2)+s(3,4) t(4,2)] \\
& M_{L R}^{c-4}=-2 i g^{2} g_{V}^{2} C_{L}^{V e} C_{R}^{V e} D_{f}\left(p_{1}-p_{3}-p_{4}\right) D_{V}\left(p_{3}+p_{4}\right) D_{W}\left(p_{2}-p_{6}\right) \\
& t(5,2) s(3,1) *[s(6,1) t(1,4)-s(6,3) t(3,4)] \\
& M_{L L}^{c-4}=-2 i g^{2} g_{V}^{2} C_{L}^{V e} C_{L}^{V e} D_{f}\left(p_{1}-p_{3}-p_{4}\right) D_{V}\left(p_{3}+p_{4}\right) D_{W}\left(p_{2}-p_{6}\right) \\
& t(5,2) s(4,1) *[s(6,1) t(1,3)-s(6,4) t(4,3)] \\
& M_{L L}^{d}=\frac{-4 i g^{2} g_{V}^{2}}{\cos ^{2} \theta_{w}} C_{L}^{Z \nu} C_{L}^{Z e} C_{L}^{V e} C_{L}^{V e} D_{f}\left(p_{1}-p_{3}-p_{4}\right) D_{V}\left(p_{3}+p_{4}\right) D_{Z}\left(p_{5}+p_{6}\right) \\
& t(2,5) s(4,1) *[s(6,1) t(1,3)-s(6,4) t(4,3)] \\
& M_{L R}^{d}=\frac{-4 i g^{2} g_{V}^{2}}{\cos ^{2} \theta_{w}} C_{L}^{Z \nu} C_{L}^{Z e} C_{L}^{V e} C_{R}^{V e} D_{f}\left(p_{1}-p_{3}-p_{4}\right) D_{V}\left(p_{3}+p_{4}\right) D_{Z}\left(p_{5}+p_{6}\right) \\
& t(2,5) s(3,1) *[s(6,1) t(1,4)-s(6,3) t(3,4)] \\
& M_{R L}^{d}=\frac{-4 i g^{2} g_{V}^{2}}{\cos ^{2} \theta_{w}} C_{L}^{Z \nu} C_{R}^{Z e} C_{R}^{V e} C_{L}^{V e} D_{f}\left(p_{1}-p_{3}-p_{4}\right) D_{V}\left(p_{3}+p_{4}\right) D_{Z}\left(p_{5}+p_{6}\right) \\
& s(2,6) t(3,1) *[t(5,1) s(1,4)-t(5,3) s(3,4)] \\
& M_{R R}^{d}=\frac{-4 i g^{2} g_{V}^{2}}{\cos ^{2} \theta_{w}} C_{L}^{Z \nu} C_{R}^{Z e} C_{R}^{V e} C_{R}^{V e} D_{f}\left(p_{1}-p_{3}-p_{4}\right) D_{V}\left(p_{3}+p_{4}\right) D_{Z}\left(p_{5}+p_{6}\right) \\
& s(2,6) t(4,1) *[t(5,1) s(1,3)-t(5,4) s(4,3)] \\
& M_{L L}^{d-2}=\frac{-4 i g^{2} g_{V}^{2}}{\cos ^{2} \theta_{w}} C_{L}^{Z \nu} C_{L}^{Z e} C_{L}^{V e} C_{L}^{V e} D_{f}\left(p_{1}-p_{5}-p_{6}\right) D_{V}\left(p_{3}+p_{4}\right) D_{Z}\left(p_{5}+p_{6}\right) \\
& s(6,1) t(2,3) *[t(1,5) s(4,1)-t(6,5) s(4,6)] \\
& M_{L R}^{d-2}=\frac{-4 i g^{2} g_{V}^{2}}{\cos ^{2} \theta_{w}} C_{L}^{Z \nu} C_{L}^{Z e} C_{L}^{V e} C_{R}^{V e} D_{f}\left(p_{1}-p_{5}-p_{6}\right) D_{V}\left(p_{3}+p_{4}\right) D_{Z}\left(p_{5}+p_{6}\right) \\
& s(6,1) t(2,4) *[t(1,5) s(3,1)-t(6,5) s(3,6)] \\
& M_{R L}^{d-2}=\frac{-4 i g^{2} g_{V}^{2}}{\cos ^{2} \theta_{w}} C_{L}^{Z \nu} C_{R}^{Z e} C_{R}^{V e} C_{L}^{V e} D_{f}\left(p_{1}-p_{5}-p_{6}\right) D_{V}\left(p_{3}+p_{4}\right) D_{Z}\left(p_{5}+p_{6}\right) \\
& t(5,1) s(2,4) *[s(1,6) * t(3,1)-s(5,6) t(3,5)] \\
& M_{R R}^{d-2}=\frac{-4 i g^{2} g_{V}^{2}}{\cos ^{2} \theta_{w}} C_{L}^{Z \nu} C_{R}^{Z e} C_{R}^{V e} C_{R}^{V e} D_{f}\left(p_{1}-p_{5}-p_{6}\right) D_{V}\left(p_{3}+p_{4}\right) D_{Z}\left(p_{5}+p_{6}\right) \\
& t(5,1) s(2,3) *[s(1,6) t(4,1)-s(5,6) t(4,5)]
\end{aligned}
$$




$$
\begin{aligned}
& M_{L L}^{e}=\frac{+4 i g^{2} g_{V}^{2}}{\cos ^{2} \theta_{w}} C_{L}^{Z \nu} C_{L}^{Z e} C_{L}^{V e} C_{L}^{V e} D_{f}\left(p_{4}+p_{5}+p_{6}\right) D_{V}\left(p_{1}+p_{2}\right) D_{Z}\left(p_{5}+p_{6}\right) \\
& t(3,2) s(6,4) *[s(1,4) t(4,5)+s(1,6) t(6,5)] \\
& M_{R L}^{e}=\frac{+4 i g^{2} g_{V}^{2}}{\cos ^{2} \theta_{w}} C_{L}^{Z \nu} C_{L}^{Z e} C_{R}^{V e} C_{L}^{V e} D_{f}\left(p_{4}+p_{5}+p_{6}\right) D_{V}\left(p_{1}+p_{2}\right) D_{Z}\left(p_{5}+p_{6}\right) \\
& t(3,1) s(6,4) *[s(2,4) t(4,5)+s(2,6) t(6,5)] \\
& M_{L R}^{e}=\frac{+4 i g^{2} g_{V}^{2}}{\cos ^{2} \theta_{w}} C_{L}^{Z \nu} C_{R}^{Z e} C_{R}^{V e} C_{L}^{V e} D_{f}\left(p_{4}+p_{5}+p_{6}\right) D_{V}\left(p_{1}+p_{2}\right) D_{Z}\left(p_{5}+p_{6}\right) \\
& s(3,1) t(5,4) *[t(2,4) s(4,6)+t(2,5) s(5,6)] \\
& M_{R R}^{e}=\frac{+4 i g^{2} g_{V}^{2}}{\cos ^{2} \theta_{w}} C_{L}^{Z \nu} C_{R}^{Z e} C_{R}^{V e} C_{R}^{V e} D_{f}\left(p_{4}+p_{5}+p_{6}\right) D_{V}\left(p_{1}+p_{2}\right) D_{Z}\left(p_{5}+p_{6}\right) \\
& s(3,2) t(5,4) *[t(1,4) s(4,6)+t(1,5) s(5,6)] \\
& M_{L L}^{e-2}=\frac{-4 i g^{2} g_{V}^{2}}{\cos ^{2} \theta_{w}} C_{L}^{Z \nu} C_{L}^{Z e} C_{L}^{V e} C_{L}^{V e} D_{f}\left(p_{3}+p_{5}+p_{6}\right) D_{V}\left(p_{1}+p_{2}\right) D_{Z}\left(p_{5}+p_{6}\right) \\
& t(3,5) s(1,4) *[s(6,3) t(3,2)+s(6,5) t(5,2)] \\
& M_{R L}^{e-2}=\frac{-4 i g^{2} g_{V}^{2}}{\cos ^{2} \theta_{w}} C_{L}^{Z \nu} C_{L}^{Z e} C_{R}^{V e} C_{L}^{V e} D_{f}\left(p_{3}+p_{5}+p_{6}\right) D_{V}\left(p_{1}+p_{2}\right) D_{Z}\left(p_{5}+p_{6}\right) \\
& t(3,5) s(2,4) *[s(6,3) t(3,1)+s(6,5) t(5,1)] \\
& M_{L R}^{e-2}=\frac{-4 i g^{2} g_{V}^{2}}{\cos ^{2} \theta_{w}} C_{L}^{Z \nu} C_{R}^{Z e} C_{R}^{V e} C_{L}^{V e} D_{f}\left(p_{3}+p_{5}+p_{6}\right) D_{V}\left(p_{1}+p_{2}\right) D_{Z}\left(p_{5}+p_{6}\right) \\
& s(3,6) t(2,4) *[t(5,3) s(3,1)+t(5,6) s(6,1)] \\
& M_{R R}^{e-2}=\frac{-4 i g^{2} g_{V}^{2}}{\cos ^{2} \theta_{w}} C_{L}^{Z \nu} C_{R}^{Z e} C_{R}^{V e} C_{R}^{V e} D_{f}\left(p_{3}+p_{5}+p_{6}\right) D_{V}\left(p_{1}+p_{2}\right) D_{Z}\left(p_{5}+p_{6}\right) \\
& s(3,6) t(1,4) *[t(5,3) s(3,2)+t(5,6) s(6,2)] \\
& M_{L L}^{f}=+4 i g_{Z}^{4} C_{L}^{Z \nu^{2}} C_{L}^{Z e^{2}} D_{f}\left(p_{3}+p_{4}+p_{6}\right) D_{Z}\left(p_{1}+p_{2}\right) D_{Z}\left(p_{3}+p_{4}\right) \\
& t(5,2) s(4,6) *[s(1,6) t(6,3)+s(1,4) t(4,3)] \\
& M_{L R}^{f}=+4 i g_{Z}^{4} C_{L}^{Z \nu^{2}} C_{L}^{Z e} C_{R}^{Z e} D_{f}\left(p_{3}+p_{4}+p_{6}\right) D_{Z}\left(p_{1}+p_{2}\right) D_{Z}\left(p_{3}+p_{4}\right) \\
& t(5,2) s(3,6) *[s(1,6) t(6,4)+s(1,3) t(3,4)] \\
& M_{R L}^{f}=+4 i g_{Z}^{4} C_{L}^{Z \nu^{2}} C_{L}^{Z e} C_{R}^{Z e} D_{f}\left(p_{3}+p_{4}+p_{6}\right) D_{Z}\left(p_{1}+p_{2}\right) D_{Z}\left(p_{3}+p_{4}\right) \\
& t(5,1) s(4,6) *[s(2,6) t(6,3)+s(2,4) t(4,3)] \\
& M_{R R}^{f}=+4 i g_{Z}^{4} C_{L}^{Z \nu^{2}} C_{L}^{Z e^{2}} D_{f}\left(p_{3}+p_{4}+p_{6}\right) D_{Z}\left(p_{1}+p_{2}\right) D_{Z}\left(p_{3}+p_{4}\right) \\
& t(5,1) s(3,6) *[s(2,6) t(6,4)+s(2,3) t(3,4)]
\end{aligned}
$$




$$
\begin{aligned}
& M_{L L}^{f-2}=-4 i g_{Z}^{4} C_{L}^{Z \nu^{2}} C_{L}^{Z e^{2}} D_{f}\left(p_{3}+p_{4}+p_{5}\right) D_{Z}\left(p_{1}+p_{2}\right) D_{Z}\left(p_{3}+p_{4}\right) \\
& t(5,3) s(1,6) *[s(4,5) t(5,2)+s(4,3) t(3,2)] \\
& M_{L R}^{f-2}=-4 i g_{Z}^{4} C_{L}^{Z \nu^{2}} C_{L}^{Z e} C_{R}^{Z e} D_{f}\left(p_{3}+p_{4}+p_{5}\right) D_{Z}\left(p_{1}+p_{2}\right) D_{Z}\left(p_{3}+p_{4}\right) \\
& t(5,4) s(1,6) *[s(3,5) t(5,2)+s(3,4) t(4,2)] \\
& M_{R L}^{f-2}=-4 i g_{Z}^{4} C_{L}^{Z \nu^{2}} C_{L}^{Z e} C_{R}^{Z e} D_{f}\left(p_{3}+p_{4}+p_{5}\right) D_{Z}\left(p_{1}+p_{2}\right) D_{Z}\left(p_{3}+p_{4}\right) \\
& t(5,3) s(2,6) *[s(4,5) t(5,1)+s(4,3) t(3,1)] \\
& M_{R R}^{f-2}=-4 i g_{Z}^{4} C_{L}^{Z \nu^{2}} C_{R}^{Z e^{2}} D_{f}\left(p_{3}+p_{4}+p_{5}\right) D_{Z}\left(p_{1}+p_{2}\right) D_{Z}\left(p_{3}+p_{4}\right) \\
& t(5,4) s(2,6) *[s(3,5) t(5,1)+s(3,4) t(4,1)] \\
& M_{L L}^{g}=-i g^{4} D_{f}\left(p_{1}-p_{3}-p_{6}\right) D_{W}\left(p_{3}+p_{6}\right) D_{W}\left(p_{4}+p_{5}\right) \\
& t(2,5) s(6,1) *[s(4,1) t(1,3)-s(4,6) t(6,3)] \\
& M_{L L}^{h}=-i g^{4} D_{f}\left(p_{3}-p_{1}+p_{5}\right) D_{W}\left(p_{1}-p_{5}\right) D_{W}\left(p_{2}-p_{6}\right) \\
& t(3,5) s(6,4) *[s(1,3) t(3,2)+s(1,5) t(5,2)] \\
& M_{L L}^{i}=-2 i g^{2} g_{V}^{2} C_{L}^{Z e} C_{L}^{Z e} D_{f}\left(p_{3}+p_{5}+p_{6}\right) D_{V}\left(p_{1}+p_{2}\right) D_{W}\left(p_{3}+p_{6}\right) \\
& t(5,3) s(1,4) *[s(6,5) t(5,2)+s(6,3) t(3,2)] \\
& M_{R L}^{i}=-2 i g^{2} g_{V}^{2} C_{R}^{Z e} C_{L}^{Z e} D_{f}\left(p_{3}+p_{5}+p_{6}\right) D_{V}\left(p_{1}+p_{2}\right) D_{W}\left(p_{3}+p_{6}\right) \\
& t(5,3) s(2,4) *[s(6,5) t(5,1)+s(6,3) t(3,1)] \\
& M_{L L}^{i-2}=+2 i g^{2} g_{V}^{2} C_{L}^{Z e} C_{L}^{Z e} D_{f}\left(p_{4}+p_{5}+p_{6}\right) D_{V}\left(p_{1}+p_{2}\right) D_{W}\left(p_{4}+p_{5}\right) \\
& t(3,2) s(4,6) *[s(1,4) t(4,5)+s(1,6) t(6,5)] \\
& M_{R L}^{i-2}=+2 i g^{2} g_{V}^{2} C_{R}^{Z e} C_{L}^{Z e} D_{f}\left(p_{4}+p_{5}+p_{6}\right) D_{V}\left(p_{1}+p_{2}\right) D_{W}\left(p_{4}+p_{5}\right) \\
& t(3,1) s(4,6) *[s(2,4) t(4,5)+s(2,6) t(6,5)] \\
& M_{L L}^{j}=+2 i g^{2} g_{Z}^{2} C_{L}^{Z \nu} C_{L}^{Z e} D_{f}\left(p_{3}+p_{4}+p_{6}\right) D_{Z}\left(p_{1}+p_{2}\right) D_{W}\left(p_{3}+p_{6}\right) \\
& t(5,2) s(6,4) *[s(1,6) t(6,3)+s(1,4) t(4,3)] \\
& M_{R L}^{j}=+2 i g^{2} g_{Z}^{2} C_{L}^{Z \nu} C_{R}^{Z e} D_{f}\left(p_{3}+p_{4}+p_{6}\right) D_{Z}\left(p_{1}+p_{2}\right) D_{W}\left(p_{3}+p_{6}\right) \\
& t(5,1) s(6,4) *[s(2,6) t(6,3)+s(2,4) t(4,3)]
\end{aligned}
$$




$$
\begin{gathered}
M_{L L}^{j-2}=-2 i g^{2} g_{Z}^{2} C_{L}^{Z \nu} C_{L}^{Z e} D_{f}\left(p_{3}+p_{4}+p_{5}\right) D_{Z}\left(p_{1}+p_{2}\right) D_{W}\left(p_{4}+p_{5}\right) \\
t(3,5) s(1,6) *[s(4,5) t(5,2)+s(4,3) t(3,2)] \\
M_{R L}^{j-2}=-2 i g^{2} g_{Z}^{2} C_{L}^{Z \nu} C_{R}^{Z e} D_{f}\left(p_{3}+p_{4}+p_{5}\right) D_{Z}\left(p_{1}+p_{2}\right) D_{W}\left(p_{4}+p_{5}\right) \\
t(3,5) s(2,6) *[s(4,5) t(5,1)+s(4,3) t(3,1)]
\end{gathered}
$$

where $c-2$ is obtained from $c$ with the $Z$ coming from the $\overline{\nu_{e}}$ leg; $c-3$ is obtained from $c$ with the $Z$ coming from the $\nu_{e}$ leg; $c-4$ is obtained from $c$ with the $\gamma, Z$ coming from the $e^{-}$leg; $d-2$ is obtained from $d$ with the $Z$ coming from the $e^{-}$leg; $e-2$ is obtained from $e$ with the $Z$ coming from the $\mu^{-}$leg; $f-2$ is obtained from $f$ with the $Z$ coming from the $\nu$ leg; $i-2$ is obtained from $i$ with the $W$ coming from the $\mu^{+}$leg; $j-2$ is obtained from $j$ with the $W$ coming from the $\nu$ leg.

The propagator denominators are defined as

$$
\begin{aligned}
D_{f}\left(p_{i}\right) & =\left(p_{i}^{2}\right)^{-1} \\
D_{W}\left(p_{i}\right) & =\left(p_{i}^{2}-M_{W}^{2}+i \Gamma_{W} M_{W}\right)^{-1} \\
D_{\gamma}\left(p_{i}\right) & =\left(p_{i}^{2}\right)^{-1} \\
D_{Z^{0}}\left(p_{i}\right) & =\left(p_{i}^{2}-M_{Z}^{2}+i \Gamma_{Z} M_{Z}\right)^{-1}
\end{aligned}
$$

and $C_{R}^{e}=\sin ^{2} \theta_{W}$ and $C_{L}^{e}=-\frac{1}{2}+\sin ^{2} \theta_{W}$. In diagrams (a) and (b) $p_{Z}$ and $p_{W^{ \pm}}$represent the gauge boson momentum flowing into the vertex. The first subscript of the amplitudes refers to the helicity of the electron and the second subscript to the helicity of the muon. To obtain the cross section the amplitudes for given electron and photon helicities are summed over and squared. These are then averaged to obtain the spin averaged matrix element squared and finally integrated over the final state phase space to yield the cross section. 


\section{REFERENCES}

[1] See for example, B. Wiik, Proceedings of the Workshop on Physics and Experiments with Linear $e^{+} e^{-}$Colliders, Waikoloa Hawaii, April 1993 (World Scientific; in press).

[2] D. Treille, Proceedings of the Workshop on Physics and Experiments with Linear $e^{+} e^{-}$ Colliders, Waikoloa Hawaii, April 1993 (World Scientific; in press); A. Djouadi and P.M. Zerwas, Proceedings of Beyond the Standard Model III, ed. S. Godfrey and P. Kayniak, Ottawa Canada, June 1992, (World Scientific, Singapore, 1993) p. 204; A. Miyamoto, Proceedings of the Workshop on Physics and Experiments at Linear $e^{+} e^{-}$Colliders, Waikoloa, Hawaii, April 26-30 1993.

[3] W. Beenakker and A. Denner, Proceedings of the Workshop on Physics and Experiments at Linear $e^{+} e^{-}$Colliders, Waikoloa, Hawaii, April 26-30 1993, CERN-TH.6928/93; H. Anlauf, A. Himmler, P. Manakos, T. Mannel, H. Dahmen, ibid; T. Ishikawa et al., KEK Preprint 92-210 (1993; unpublished).

[4] A. Aeppli, F. Cuypers, and G.J. van Oldenborgh, Paul Scherrer Institut report PSIPR-93-05;

[5] G. Couture, S. Godfrey, P. Kalyniak Phys. Rev D39, 3239 (1989), Phys. Rev. D42,1841 (1990), Phys. Lett. B218, 361 (1989) and references therein.

[6] P. Kalyniak, P. Madsen, N. Sinha, and R. Sinha, Phys. Rev. D48,5081 (1993).

[7] G. Couture, S. Godfrey, and R. Lewis, Phys. Rev. D45, 777 (1992).

[8] E. Yehudai, Phys. Rev. D 41, 33 (1990); D44, 3434 (1991); S. Godfrey and A.K. Peterson, Carleton University report OCIP/C-92-7.

[9] K. Hagiwara et al., Nucl. Phys. B365, 544 (1991) and references therein.

[10] S. Ambrosanio and B. Mele, Nucl. Phys. B374, 3 (1992).

[11] M. Swartz, Invited talk at the XVI International Symposium on Lepton-Photon Inter- 
actions, Cornell University, Ithaca New York, August 10-15, 1993.

[12] W. Hollik, Invited talk at the XVI International Symposium on Lepton-Photon Interactions, Cornell University, Ithaca New York, August 10-15, 1993.

[13] C.P. Burgess, S. Godfrey, H. König, D. London, and I. Maksymyk, Carleton University report OCIP/C-93-6 (1993; unpublished).

[14] S.L. Glashow, Nucl. Phys. 22, 579 (1961); S. Weinberg, Phys. Rev. Lett. 19, 1264 (1967); A. Salam, Proc. of the 8th Nobel Symposium, ed. N. Svartholm (Willey, New York 1968).

[15] D. Zeppenfeld, Phys. Let. 183B, 380 (1987); D. Treille et al, Proceedings of the ECFA Workshop on LEP 200, ed. A. Böhm and W. Hoogland, Aachen (1986), CERN 87-08, vol.2, p.414. D.A. Dicus, K. Kallianpur, Phys. Rev. D32, 35 (1985); M.J. Duncan, G.L. Kane, Phys. Rev. Lett. 55, 773 (1985).

[16] Proceedings of the First Workshop on Japan Linear Collider (JLC), KEK, Oct 24-25, 1989, KEK Report 90-2 (1990); Proceedings of the Second Workshop on Japan Linear Collider, ed. S. Kawabata, Nov. 6-8, 1990 KEK Proceedings 91-10 Nov. 1991.

[17] C. Ahn et al., Opportunities and Requirments for Experimentation at a Very High Energy $e^{+} e^{-}$Collider, SLAC Report SLAC-0329 (1988);

[18] Proceedings of the Workshop on Physics at Future Accelerators, CERN Yellow Report 87-07 (1987).

[19] R. J. Noble, Nucl. Instrum. Methods A256, 427 (1987); R. Blankenbecler and S.D. Drell, Phys. Rev. D36, 277 (1987); D37, 3308 (1988); Phys. Rev. Lett. 61, 2324 (1988); R. Blankenbecler, S.D. Drell and N. Kroll, Phys. Rev. D40, 2462 (1989); D. V. Schroeder, PhD Thesis, SLAC Report SLAC-371 (1990, unpublished).

[20] S.Y. Choi and F. Schrempp, Phys. Lett. 272B, 149 (1991). 
[21] J. Bagger, Proceedings of the 1991 TASI Institute, (World Scientific, Singapore, 1992).

[22] K. Hagiwara et al, Nucl. Phys. B282, 253 (1987).

[23] K. Gaemers and G. Gounaris, Z. Phys. C1, 259 (1979).

[24] K.-i Hikasa Phys. Rev. D33, 3203 (1986); K. Hagiwara et al, Nucl. Phys. B282, 253 (1987); J.Fleischer et al. Z. Phys., C42, 409 (1989).

[25] A. de Rújula, M.B. Gavela, P. Hernandez and E. Massó, Nucl. Phys. B384, 3(1993).

[26] C.P. Burgess and D. London, Phys. Rev. D48, 4337(1993); Phys. Rev. Lett. 69, 3428 (1992).

[27] A.F. Falk, M. Luke, and E.H. Simmons, Nucl. Phys. B365, 523 (1991); D. Zeppenfeld, Proceedings of the Workshop on Physics and Experiments at Linear $e^{+} e^{-}$Colliders, Waikoloa, Hawaii, April 26-30 1993.

[28] W.J. Marciano, A. Queijeiro, Phys. Rev. D 33, 3449 (1986); F. Boudjema, K. Hagiwara, C. Hamzaoui, and K. Numata, Phys. Rev. D43, 2223 (1991).

[29] G. Couture, J.N. Ng, Z.Phys. C35, 65(1987); G. Couture et al, Phys.Rev. D38, 860 (1988); Phys.Rev. D36, 859 (1987) and references therein.

[30] C.P. Burgess, S. Godfrey, H. König, D. London, and I. Maksymyk, Carleton University report OCIP/C-93-7 (1993; unpublished) (hepph-9307223).

[31] K. Hagiwara, et al. Phys. Lett. B283, 353 (1992).

[32] P. Hernández and F.J. Vegas, Phys. Lett. B307, 116(1993); D. Choudhury, P. Roy and R. Sinha, preprint TIFR-TH/93-08 (unpublished).

[33] J. Alitti et al., UA2 Collaboration, CERN Report CERN-PPE/92-216 (1991).

[34] M.S. Samuel et al, Phys. Rev. Lett. 67, 9 (1991); M. Samuel et al, Phys. Rev. D44, 2064 (1991). 
[35] U. Baur and E. L. Berger, Phys. Rev. D41, 1476 (1990) and references therein.

[36] D. Atwood et al., W Production in ep Collisions, Proceedings of the 1988 Snowmass Summer Study High Energy Physics in the 1990's, p. 264; U. Baur and D. Zeppenfeld, Nucl. Phys. B325, 253 (1989).

[37] D. Atwood et al, $W$ Production at ep Colliders in the Process ep $\rightarrow W^{-} \nu+X$, Proceedings of the 1990 Summer Study on Research Directions for the Decade, Snowmass, Colorado, June 25 - July 13, 1990, University of Wisconsin preprint MAD/PH/591 (1990).

[38] P. Salati and J.C. Wallet, Z. Phys. C16, 155 (1982); A.N. Kamal et al., Phys. Rev. D24, 2482 (1984); H. Neufeld, Z. Phys. C17, 145 (1983); G. Altarelli et al., Nucl. Phys. B262, 204 (1985); E. Gabrielli, Mod. Phys. Lett. A1, 465 (1986); M. Böhm and A. Rosado, Z. Phys. C39 275 (1988).

[39] S. Godfrey, Z. Phys. C55, 619(1992); U. Baur and M.A. Doncheski, University of Wisconsin report MAD/PH/692, (1992; unpublished); T. Helbig and H. Spiesberger, Nucl. Phys. B373, 73 (1992).

[40] G.Kane, J. Vidal, C.P. Yuan, Phys. Rev. D39, 2617 (1990), and references therein.

[41] G. Couture, Phys. Rev. D44, 2755 (1991).

[42] U. Baur, D. Zeppenfeld, Nucl. Phys. B308, 127 (1988); D. Zeppenfeld and S. Willenbrock, Phys. Rev. D37, 1775 (1988); U. Baur, D. Zeppenfeld, Phys. Lett. 201B, 383 (1988); F. Patore and M. Pepe, Proceedings of the Large Hadron Collider Workshop, Aachen, October 4-9 1990, CERN 90-10 Vol II p. 106.

[43] G. Bélanger, F. Boudjema, and D. London, Phys. Rev. Lett. 65, 2943 (1990)

[44] H. Iwasaki, Hiroshima University report HUPD-9106 (1991).

[45] R. Kleiss and W. J. Stirling, Nucl. Phys. B262, 235 (1985); Z.Xu D.-H.Zhang L. Chang 
Nucl. Phys. B291, 392 (1987).

[46] See for example V. Barger and R. Phillips, Collider Physics, (Addison-Wesley Publishing Company, 1987).

[47] M. Kuroda, F.M. Renard, D. Schildknecht, Phys. Lett. B183, 366 (1987).

[48] T. Barklow, private communication. 


\section{FIGURES}

FIG. 1. The trilinear Gauge Boson Vertex

FIG. 2. The Feynman diagrams contributing to the process $e^{+} e^{-} \rightarrow \mu^{+} \mu^{-} \nu \bar{\nu}$

FIG. 3. The cross section $\sigma\left(e^{+} e^{-} \rightarrow \mu^{+} \mu^{-} \nu \bar{\nu}\right)$ as a function of $\sqrt{s}$. The solid line is for the cuts on $E_{\mu^{ \pm}}, \not p_{T}$, and $\cos \theta_{\mu^{ \pm}}$given in the text. The dashed line adds the cut $M_{\mu^{+} \mu^{-}}>25 \mathrm{GeV}$ and the dotted line has $\left|M_{\mu^{+} \mu^{-}}-M_{Z}\right|<5 \mathrm{GeV}$.

FIG. 4. (a) $d \sigma / d M_{\mu^{+} \mu_{-}}$and (b) $d \sigma / d \cos \theta_{\mu^{+} \mu^{-}}$at $\sqrt{s}=200 \mathrm{GeV}$. In both cases the solid line is for standard model values of $\kappa_{\gamma}, \lambda_{\gamma}, \kappa_{Z}$, and $\lambda_{Z}$, the long dashed line is for $\delta \kappa_{\gamma}=\lambda_{\gamma}=\lambda_{Z}=0$ and $\delta \kappa_{Z}=2$; the dotted line is for $\delta \kappa_{\gamma}=\lambda_{\gamma}=\delta \kappa_{Z}=0$; and $\lambda_{Z}=2$ the dot-dashed line is for $\delta \kappa_{Z}=\lambda_{\gamma}=\lambda_{Z}=0$ and $\delta \kappa_{\gamma}=2$ and dot-dot-dashed line is for $\delta \kappa_{\gamma}=\lambda_{Z}=\delta \kappa_{Z}=0$ and $\lambda_{\gamma}=2$ where $\delta \kappa_{V}=\kappa_{V}-1$. In this figure and all subsequent ones, the small bumps are to due statistical fluctuations arising from the Monte-Carlo phase space integration.

FIG. 5. Sensitivities of the TGV's to anomalous couplings at 95\% C.L. based on the kinematic cuts given in the text. The solid lines are based on the statistics assuming an integrated luminosity of $250 \mathrm{pb}^{-1}$ and the dashed lines are based on integrated luminosities of $500 \mathrm{pb}^{-1}$.

FIG. 6. $d \sigma / d M_{\mu^{+} \mu_{-}}$at $\sqrt{s}=500 \mathrm{GeV}$. The solid line is for standard model values of $\kappa_{\gamma}, \lambda_{\gamma}$, $\kappa_{Z}$, and $\lambda_{Z}$, the long dashed line is for $\delta \kappa_{\gamma}=\lambda_{\gamma}=\lambda_{Z}=0$ and $\delta \kappa_{Z}=-0.5$; the dotted line is for $\delta \kappa_{\gamma}=\lambda_{\gamma}=\delta \kappa_{Z}=0$ and $\lambda_{Z}=1$ the dot-dashed line is for $\delta \kappa_{Z}=\lambda_{\gamma}=\lambda_{Z}=0$ and $\delta \kappa_{\gamma}=0.5$ and dot-dot-dashed line is for $\delta \kappa_{\gamma}=\lambda_{Z}=\delta \kappa_{Z}=0$ and $\lambda_{\gamma}=0.5$.

FIG. 7. (a) $d \sigma / d \cos \theta_{\mu^{+} \mu^{-}}$and (b) $d \sigma / d p_{T Z}$ at $\sqrt{s}=500 \mathrm{GeV}$ with $\left|M_{\mu^{+} \mu^{-}}-M_{Z}\right|<5 \mathrm{GeV}$. In both cases the solid line is for standard model values of $\kappa_{\gamma}, \lambda_{\gamma}, \kappa_{Z}$, and $\lambda_{Z}$, the long dashed line is for $\delta \kappa_{\gamma}=\lambda_{\gamma}=\lambda_{Z}=0$ and $\delta \kappa_{Z}=-0.5$; the dotted line is for $\delta \kappa_{\gamma}=\lambda_{\gamma}=\delta \kappa_{Z}=0$ and $\lambda_{Z}=0.5$ and dot-dashed line is for $\delta \kappa_{\gamma}=\lambda_{Z}=\delta \kappa_{Z}=0$ and $\lambda_{\gamma}=-0.5$. 
FIG. 8. Sensitivities of the TGV's to anomalous couplings for $\sqrt{s}=500 \mathrm{GeV}$ and $\mathrm{L}=10 \mathrm{fb}^{-1}$ based on (a) $d \sigma / d \cos \theta_{\mu^{+} \mu^{-}}$(b) $d \sigma / d p_{T Z}$ with $\left|M_{\mu^{+} \mu^{-}}-M_{Z}\right|<5 \mathrm{GeV}$ using the binning given in the text. In both cases the solid lines are $68 \%$ C.L., the dashed lines are 90\% C.L., and the dot-dashed curves are 95\% C.L..

FIG. 9. $95 \%$ C.L. bounds of the TGV's based on (a) $d \sigma / d \cos \theta_{\mu^{+} \mu^{-}}$(b) $d \sigma / d p_{T Z}$ at $\sqrt{s}=500 \mathrm{GeV}$ with $\left|M_{\mu^{+} \mu^{-}}-M_{Z}\right|<5 \mathrm{GeV}$ using the binning given in the text. In both cases the solid curves are based on $10 \mathrm{fb}^{-1}$, the dashed curves on $50 \mathrm{fb}^{-1}$, the dot-dashed curves on $10 \mathrm{fb}^{-1}+\delta^{\text {sys }}$, and the dotted curves on $50 \mathrm{fb}^{-1}+\delta^{\text {sys }}$ where $\delta^{\text {sys }}=5 \%$.

FIG. 10. Sensitivities of the TGV's to anomalous couplings for $\sqrt{s}=500 \mathrm{GeV}$ and $\mathrm{L}=10 \mathrm{fb}^{-1}$ based on the total cross section integrated over the kinematic region $110 \leq M_{\mu^{+} \mu^{-}} \leq 400 \mathrm{GeV}$. The solid lines are $68 \%$ C.L., the dashed lines are $90 \%$ C.L., and the dot-dashed curves are $95 \%$ C.L..

FIG. 11. Sensitivities of the TGV's to anomalous couplings for $\sqrt{s}=1 \mathrm{TeV}$ and $\mathrm{L}=50 \mathrm{fb}^{-1}$ based on (a) $d \sigma / d \cos \theta_{\mu^{+} \mu^{-}}$(b) $d \sigma / d p_{T Z}$ with $\left|M_{\mu^{+} \mu^{-}}-M_{Z}\right|<5 \mathrm{GeV}$ using the binning given in the text. In both cases the solid lines are $68 \%$ C.L., the dashed lines are 90\% C.L., and the dot-dashed curves are 95\% C.L..

FIG. 12. $d \sigma / d M_{\mu^{+} \mu_{-}}$at $\sqrt{s}=1 \mathrm{TeV}$. The solid line is for standard model values of $\kappa_{\gamma}, \lambda_{\gamma}$, $\kappa_{Z}$, and $\lambda_{Z}$, the long dashed line is for $\delta \kappa_{\gamma}=\lambda_{\gamma}=\lambda_{Z}=0$ and $\delta \kappa_{Z}=-0.2$; the dotted line is for $\delta \kappa_{Z}=\lambda_{\gamma}=\lambda_{Z}=0$ and $\delta \kappa_{\gamma}=0.2$ and dot-dashed line is for $\delta \kappa_{\gamma}=\lambda_{Z}=\delta \kappa_{Z}=0$ and $\lambda_{\gamma}=-0.2$.

FIG. 13. Sensitivities of the TGV's to anomalous couplings for $\sqrt{s}=1 \mathrm{TeV}$ and $\mathrm{L}=50 \mathrm{fb}^{-1}$ based on the total cross section integrated over the kinematic region $200 \leq M_{\mu^{+} \mu^{-}} \leq 900 \mathrm{GeV}$. The solid lines are $68 \%$ C.L., the dashed lines are 90\% C.L., and the dot-dashed curves are $95 \%$ C.L.. 


\section{TABLES}

TABLE I. Sensitivities to $\kappa_{\gamma}, \lambda_{\gamma} \kappa_{Z}$, and $\lambda_{Z}$ at $95 \%$ C.L. from the process $e^{+} e^{-} \rightarrow \mu^{+} \mu^{-} \nu \bar{\nu}$ at a $500 \mathrm{GeV} e^{+} e^{-}$collider. The statistical error is based on the specified integrated luminosity and $\delta^{\text {sys }}$ refers to the systematic error which we take as $5 \%$.

\begin{tabular}{|c|c|c|c|c|}
\hline \multicolumn{5}{|c|}{ Based on $\sigma\left(e^{+} e^{-} \rightarrow \mu^{+} \mu^{-} \nu \bar{\nu}\right)$ with $\left|M_{\mu^{+} \mu^{-}}-M_{Z}\right|<5 \mathrm{GeV}$} \\
\hline & $\mathrm{L}=20 \mathrm{fb}^{-1}$ & $\mathrm{~L}=50 \mathrm{fb}^{-1}$ & $\mathrm{~L}=20 \mathrm{fb}^{-1}+\delta^{s y s}$ & $\mathrm{~L}=50 \mathrm{fb}^{-1}+\delta^{s y s}$ \\
\hline$\delta \kappa_{Z}$ & \pm 0.1 & \pm 0.06 & $\begin{array}{l}+0.25 \\
-0.30\end{array}$ & $\begin{array}{l}+0.24 \\
-0.28\end{array}$ \\
\hline$\delta \lambda_{Z}$ & $\begin{array}{l}+0.18 \\
-0.53 \\
\end{array}$ & $\begin{array}{l}+0.12 \\
-0.48 \\
\end{array}$ & $\begin{array}{l}+0.34 \\
-0.7 \\
\end{array}$ & $\begin{array}{l}+0.33 \\
-0.7 \\
\end{array}$ \\
\hline \multicolumn{5}{|c|}{ Based on $d \sigma / d p_{T} Z$ with $\left|M_{\mu^{+} \mu^{-}}-M_{Z}\right|<5 \mathrm{GeV}$} \\
\hline$\delta \kappa_{Z}$ & $\begin{array}{l}+0.19 \\
-0.23\end{array}$ & \pm 0.09 & $\begin{array}{l}+0.26 \\
-0.32\end{array}$ & $\begin{array}{l}+0.19 \\
-0.23\end{array}$ \\
\hline$\delta \lambda_{Z}$ & $\begin{array}{l}+0.27 \\
-0.36 \\
\end{array}$ & $\begin{array}{l}+0.15 \\
-0.20 \\
\end{array}$ & $\begin{array}{l}+0.33 \\
-0.43 \\
\end{array}$ & $\begin{array}{l}+0.27 \\
-0.38 \\
\end{array}$ \\
\hline \multicolumn{5}{|c|}{ Based on binning $M_{\mu^{+} \mu^{-}}$} \\
\hline & $\mathrm{L}=10 \mathrm{fb}^{-1}$ & $\mathrm{~L}=50 \mathrm{fb}^{-1}$ & $\mathrm{~L}=10 \mathrm{fb}^{-1}+\delta^{\text {sys }}$ & $\mathrm{L}=50 \mathrm{fb}^{-1}+\delta^{s y s}$ \\
\hline$\delta \kappa_{Z}$ & $\begin{array}{l}+0.13 \\
-0.09\end{array}$ & $\begin{array}{l}+0.08 \\
-0.05\end{array}$ & $\begin{array}{l}+0.15 \\
-0.12\end{array}$ & $\begin{array}{l}+0.13 \\
-0.10\end{array}$ \\
\hline$\delta \lambda_{Z}$ & $\begin{array}{l}+0.082 \\
-0.090\end{array}$ & $\begin{array}{l}+0.055 \\
-0.060\end{array}$ & $\begin{array}{l}+0.096 \\
-0.107\end{array}$ & $\begin{array}{l}+0.082 \\
-0.090\end{array}$ \\
\hline$\delta \kappa_{\gamma}$ & $\begin{array}{l}+0.21 \\
-0.09\end{array}$ & $\begin{array}{l}+0.17 \\
-0.05\end{array}$ & $\begin{array}{l}+0.27 \\
-0.11\end{array}$ & $\begin{array}{l}+0.21 \\
-0.08\end{array}$ \\
\hline$\delta \lambda_{\gamma}$ & $\begin{array}{l}+0.09 \\
-0.12\end{array}$ & $\begin{array}{l}+0.06 \\
-0.07\end{array}$ & $\begin{array}{l}+0.12 \\
-0.14\end{array}$ & $\begin{array}{l}+0.09 \\
-0.12\end{array}$ \\
\hline
\end{tabular}


TABLE II. Sensitivities to $\kappa_{\gamma}, \lambda_{\gamma} \kappa_{Z}$, and $\lambda_{Z}$ at $95 \%$ C.L. from the process $e^{+} e^{-} \rightarrow \mu^{+} \mu^{-} \nu \bar{\nu}$ at a $1 \mathrm{TeV} e^{+} e^{-}$collider. The statistical error is based on the specified integrated luminosity and $\delta^{\text {sys }}$ refers to the systematic error which we take as $5 \%$.

\begin{tabular}{|c|c|c|c|c|}
\hline & $\mathrm{L}=50 \mathrm{fb}^{-1}$ & $\mathrm{~L}=200 \mathrm{fb}^{-1}$ & $\mathrm{~L}=50 \mathrm{fb}^{-1}+\delta^{\text {sys }}$ & $\mathrm{L}=200 \mathrm{fb}^{-1}+\delta^{\text {sys }}$ \\
\hline \multicolumn{5}{|c|}{ Based on $d \sigma / d \cos \theta_{\mu^{+} \mu^{-}}$with $\left|M_{\mu^{+} \mu^{-}}-M_{Z}\right|<5 \mathrm{GeV}$} \\
\hline$\delta \kappa_{Z}$ & \pm 0.07 & \pm 0.03 & $\begin{array}{l}+0.13 \\
-0.16\end{array}$ & $\begin{array}{l}+0.11 \\
-0.13\end{array}$ \\
\hline$\delta \lambda_{Z}$ & $\begin{array}{l}+0.07 \\
-0.14 \\
\end{array}$ & $\begin{array}{l}+0.04 \\
-0.06 \\
\end{array}$ & $\begin{array}{l}+0.12 \\
-0.25\end{array}$ & $\begin{array}{l}+0.10 \\
-0.16 \\
\end{array}$ \\
\hline \multicolumn{5}{|c|}{ Based on $d \sigma / d p_{T} Z$ with $\left|M_{\mu^{+} \mu^{-}}-M_{Z}\right|<5 \mathrm{GeV}$} \\
\hline$\delta \kappa_{Z}$ & \pm 0.065 & \pm 0.03 & $\begin{array}{l}+0.17 \\
-0.19\end{array}$ & $\begin{array}{l}+0.16 \\
-0.18\end{array}$ \\
\hline$\delta \lambda_{Z}$ & $\begin{array}{l}+0.08 \\
-0.10 \\
\end{array}$ & $\begin{array}{l}+0.040 \\
-0.055 \\
\end{array}$ & \pm 0.18 & \pm 0.17 \\
\hline \multicolumn{5}{|c|}{ Based on $200<M_{\mu^{+} \mu^{-}}<900 \mathrm{GeV}$} \\
\hline$\delta \kappa_{Z}$ & ${ }_{-0.012}^{+0.030}$ & $\stackrel{+0.025}{-0.007}$ & $\begin{array}{l}+0.038 \\
-0.018\end{array}$ & ${ }_{-0.016}^{+0.034}$ \\
\hline$\delta \lambda_{Z}$ & $\begin{array}{l}+0.021 \\
-0.011\end{array}$ & $\begin{array}{l}+0.017 \\
-0.007\end{array}$ & $\begin{array}{l}+0.026 \\
-0.025\end{array}$ & \pm 0.024 \\
\hline$\delta \kappa_{\gamma}$ & $\begin{array}{l}+0.044 \\
-0.011\end{array}$ & $\begin{array}{l}+0.008 \\
-0.007\end{array}$ & $\begin{array}{l}+0.048 \\
-0.016\end{array}$ & $\begin{array}{l}+0.046 \\
-0.014\end{array}$ \\
\hline$\delta \lambda_{\gamma}$ & $\begin{array}{l}+0.029 \\
-0.009\end{array}$ & $\begin{array}{l}+0.025 \\
-0.006\end{array}$ & $\begin{array}{l}+0.033 \\
-0.014\end{array}$ & $\begin{array}{l}+0.032 \\
-0.012\end{array}$ \\
\hline$\delta \kappa_{Z}$ & $\begin{array}{l}+0.030 \\
-0.012\end{array}$ & $\begin{array}{l}+0.025 \\
-0.007\end{array}$ & $\begin{array}{l}+0.038 \\
-0.018\end{array}$ & $\begin{array}{l}+0.034 \\
-0.016\end{array}$ \\
\hline$\delta \lambda_{Z}$ & $\begin{array}{l}+0.021 \\
-0.011\end{array}$ & $\begin{array}{l}+0.017 \\
-0.007\end{array}$ & $\begin{array}{l}+0.026 \\
-0.025\end{array}$ & \pm 0.024 \\
\hline$\delta \kappa_{\gamma}=\delta \kappa_{Z}$ & $\begin{array}{l}+0.027 \\
-0.006\end{array}$ & $\begin{array}{l}+0.006 \\
-0.003\end{array}$ & $\begin{array}{l}+0.033 \\
-0.009\end{array}$ & $\begin{array}{l}+0.032 \\
-0.008\end{array}$ \\
\hline$\delta \lambda_{\gamma}=\delta \lambda_{Z}$ & $\begin{array}{l}+0.008 \\
-0.006\end{array}$ & \pm 0.004 & $\begin{array}{l}+0.022 \\
-0.009\end{array}$ & $\begin{array}{l}+0.021 \\
-0.008\end{array}$ \\
\hline
\end{tabular}


This figure "fig1-1.png" is available in "png" format from: http://arxiv.org/ps/hep-ph/9401306v1 


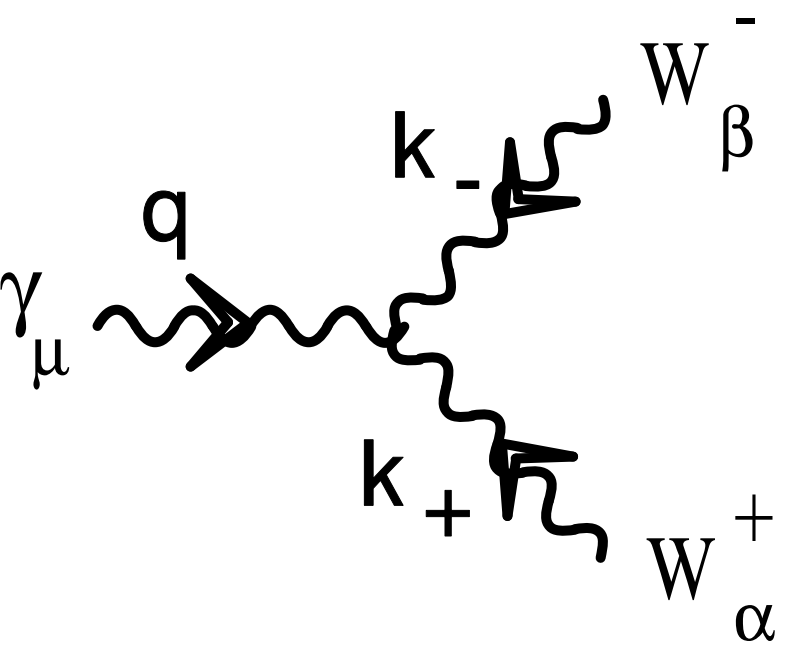

This figure "fig2-1.png" is available in "png" format from: http://arxiv.org/ps/hep-ph/9401306v1 
This figure "fig3-1.png" is available in "png" format from: http://arxiv.org/ps/hep-ph/9401306v1 
This figure "fig4-1.png" is available in "png" format from: http://arxiv.org/ps/hep-ph/9401306v1 
This figure "fig5-1.png" is available in "png" format from: http://arxiv.org/ps/hep-ph/9401306v1 
This figure "fig1-2.png" is available in "png" format from: http://arxiv.org/ps/hep-ph/9401306v1 
This figure "fig2-2.png" is available in "png" format from: http://arxiv.org/ps/hep-ph/9401306v1 

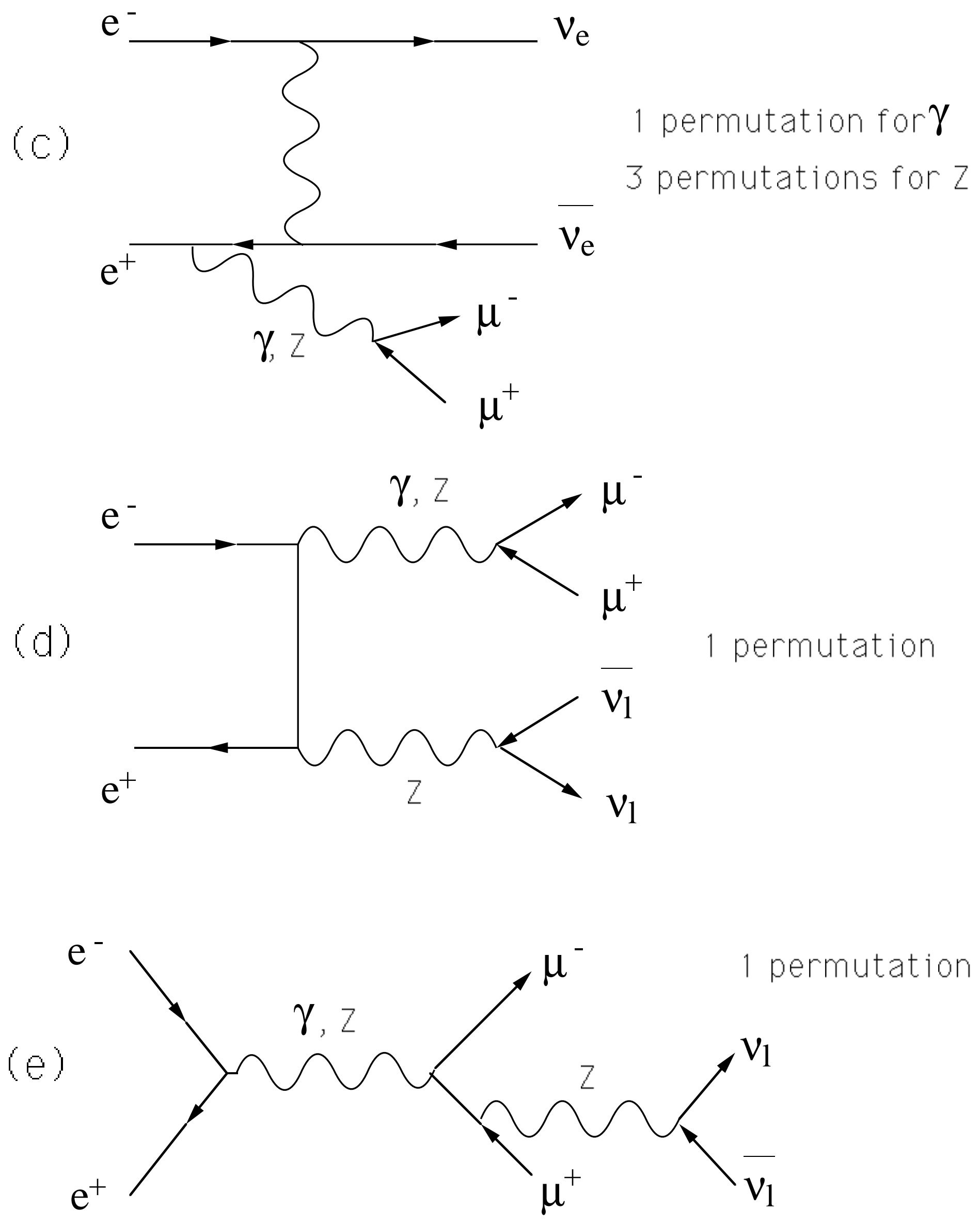

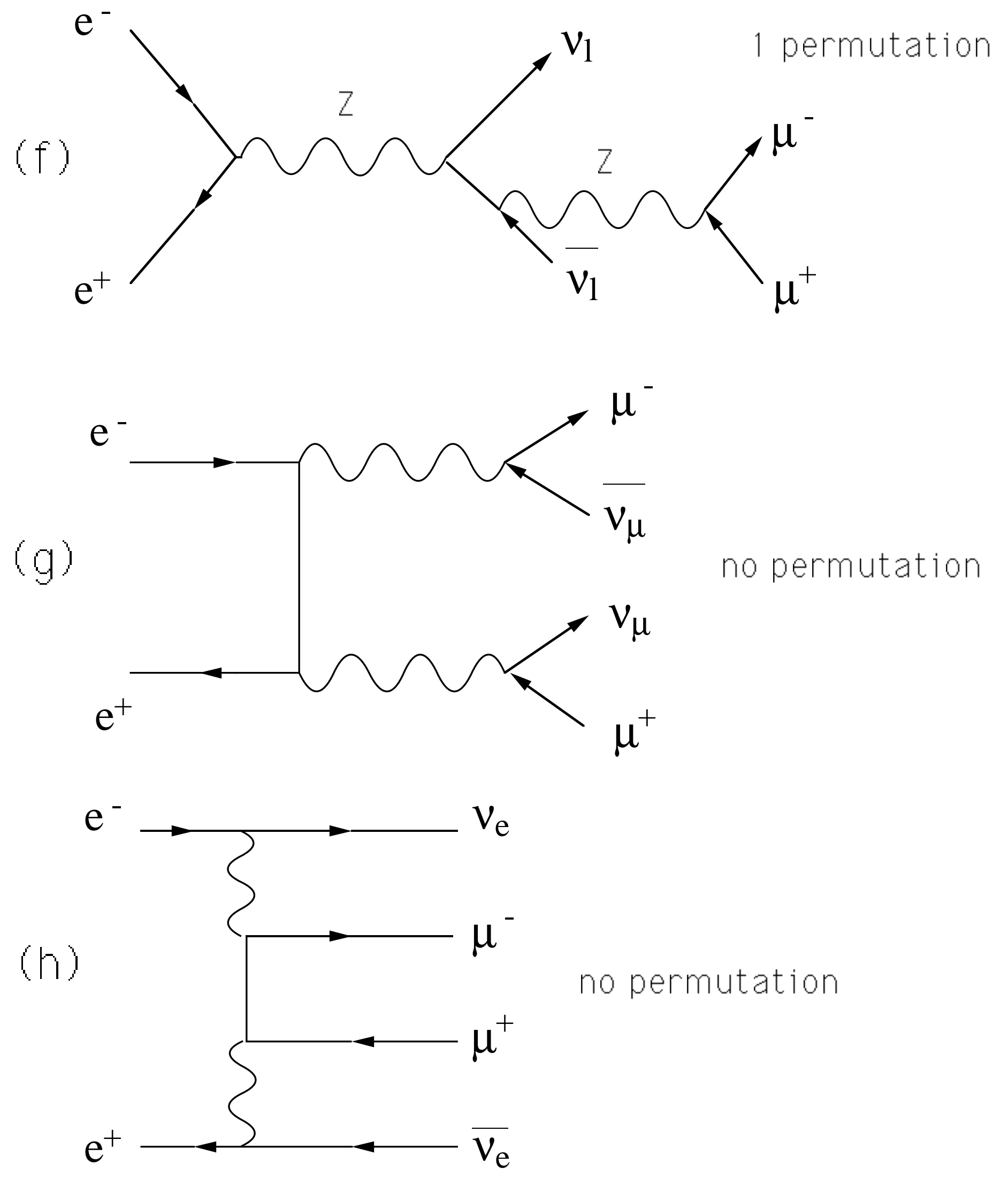

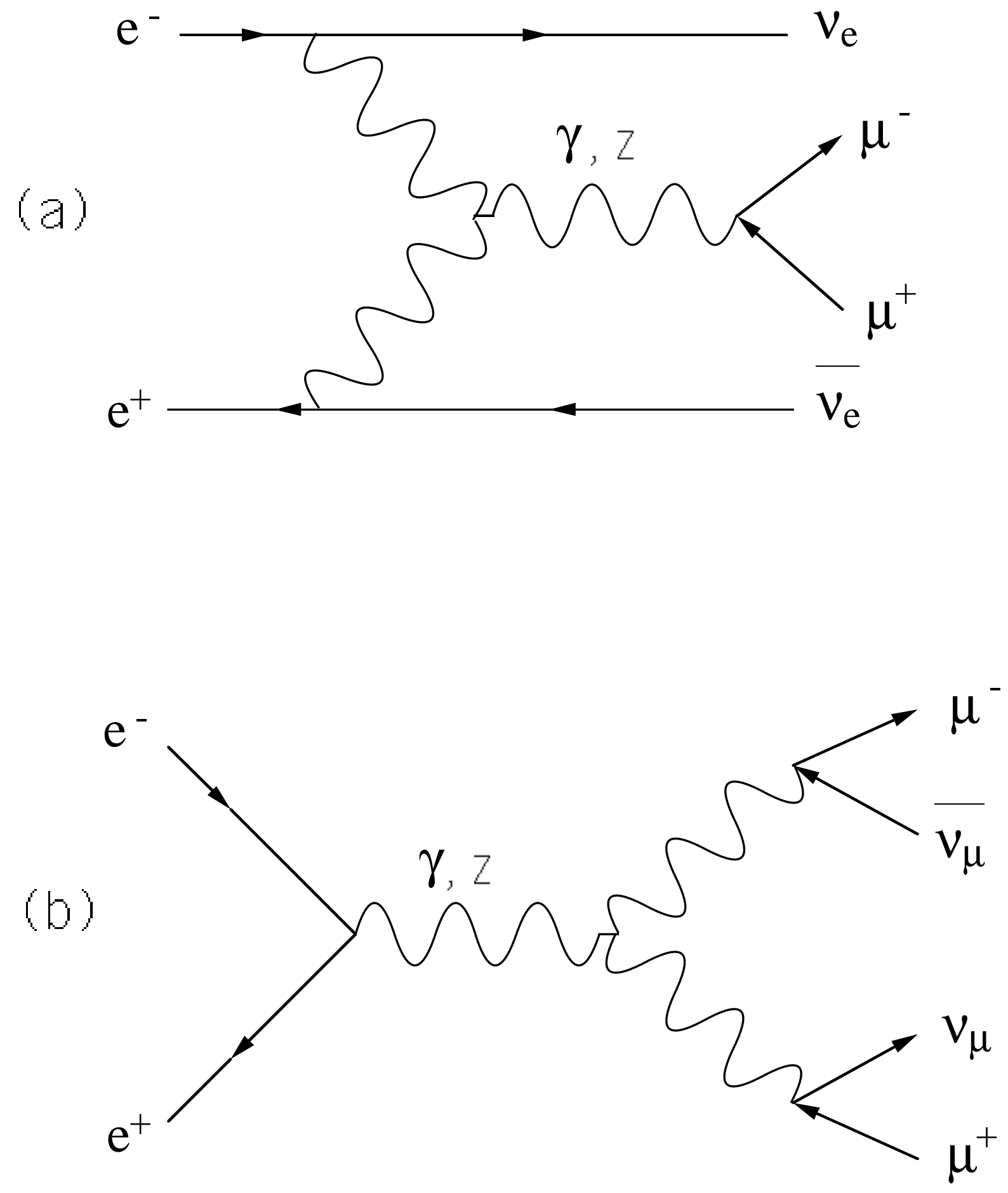

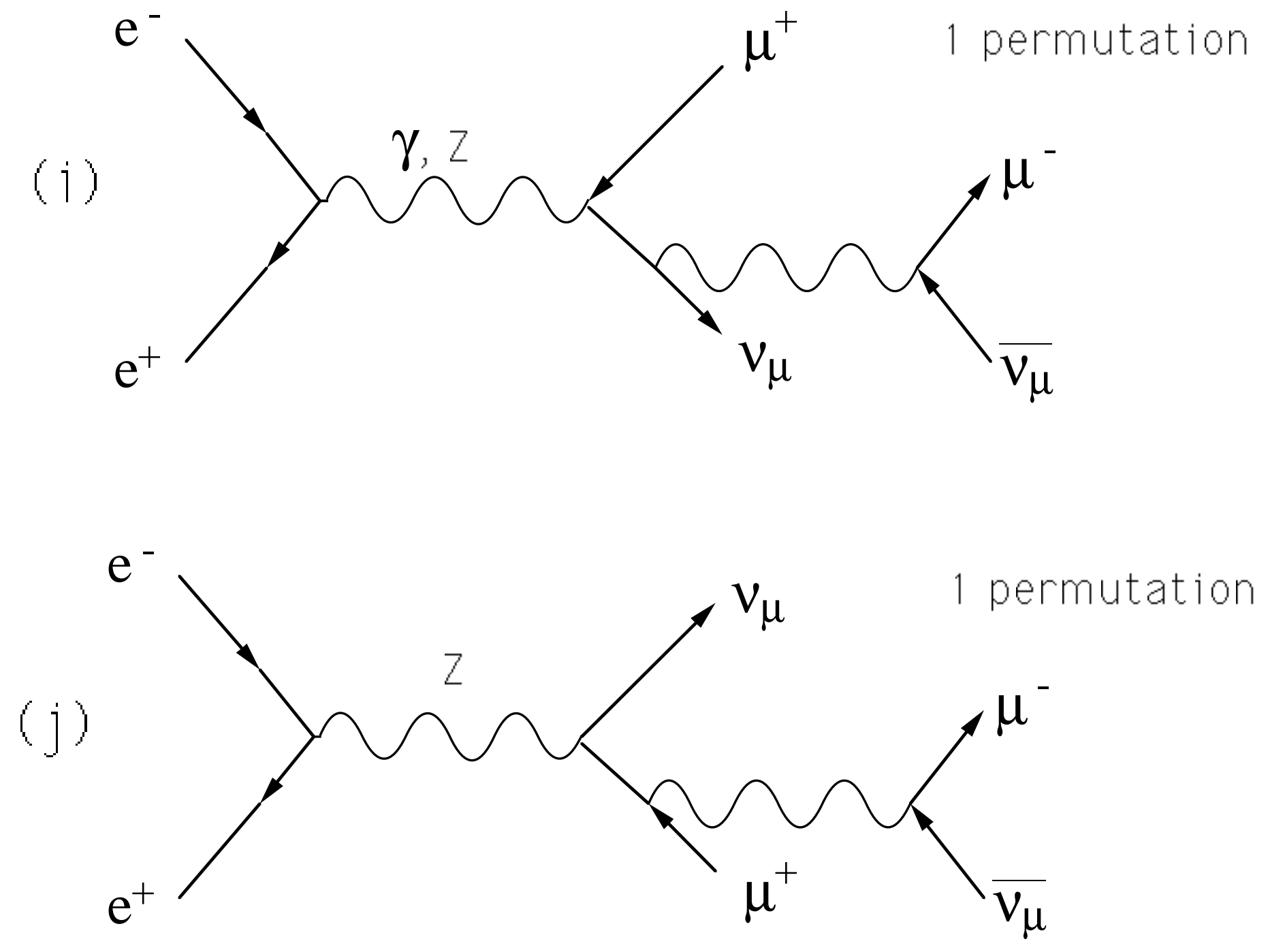
This figure "fig3-2.png" is available in "png" format from: http://arxiv.org/ps/hep-ph/9401306v1 
This figure "fig4-2.png" is available in "png" format from: http://arxiv.org/ps/hep-ph/9401306v1 
This figure "fig5-2.png" is available in "png" format from: http://arxiv.org/ps/hep-ph/9401306v1 
This figure "fig2-3.png" is available in "png" format from: http://arxiv.org/ps/hep-ph/9401306v1 
This figure "fig3-3.png" is available in "png" format from: http://arxiv.org/ps/hep-ph/9401306v1 


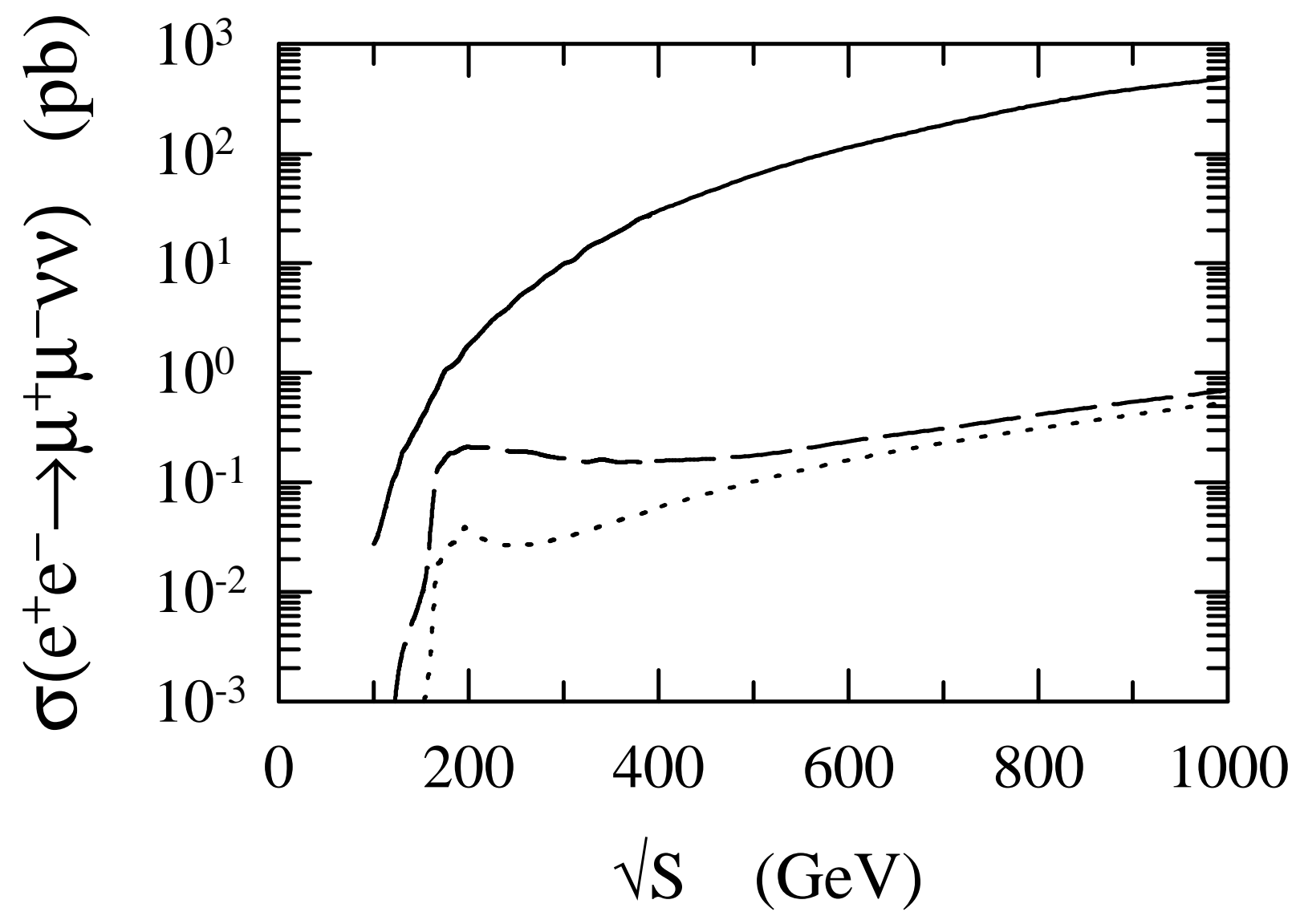


This figure "fig4-3.png" is available in "png" format from: http://arxiv.org/ps/hep-ph/9401306v1 
This figure "fig5-3.png" is available in "png" format from: http://arxiv.org/ps/hep-ph/9401306v1 
This figure "fig2-4.png" is available in "png" format from: http://arxiv.org/ps/hep-ph/9401306v1 
This figure "fig3-4.png" is available in "png" format from: http://arxiv.org/ps/hep-ph/9401306v1 
This figure "fig4-4.png" is available in "png" format from: http://arxiv.org/ps/hep-ph/9401306v1 


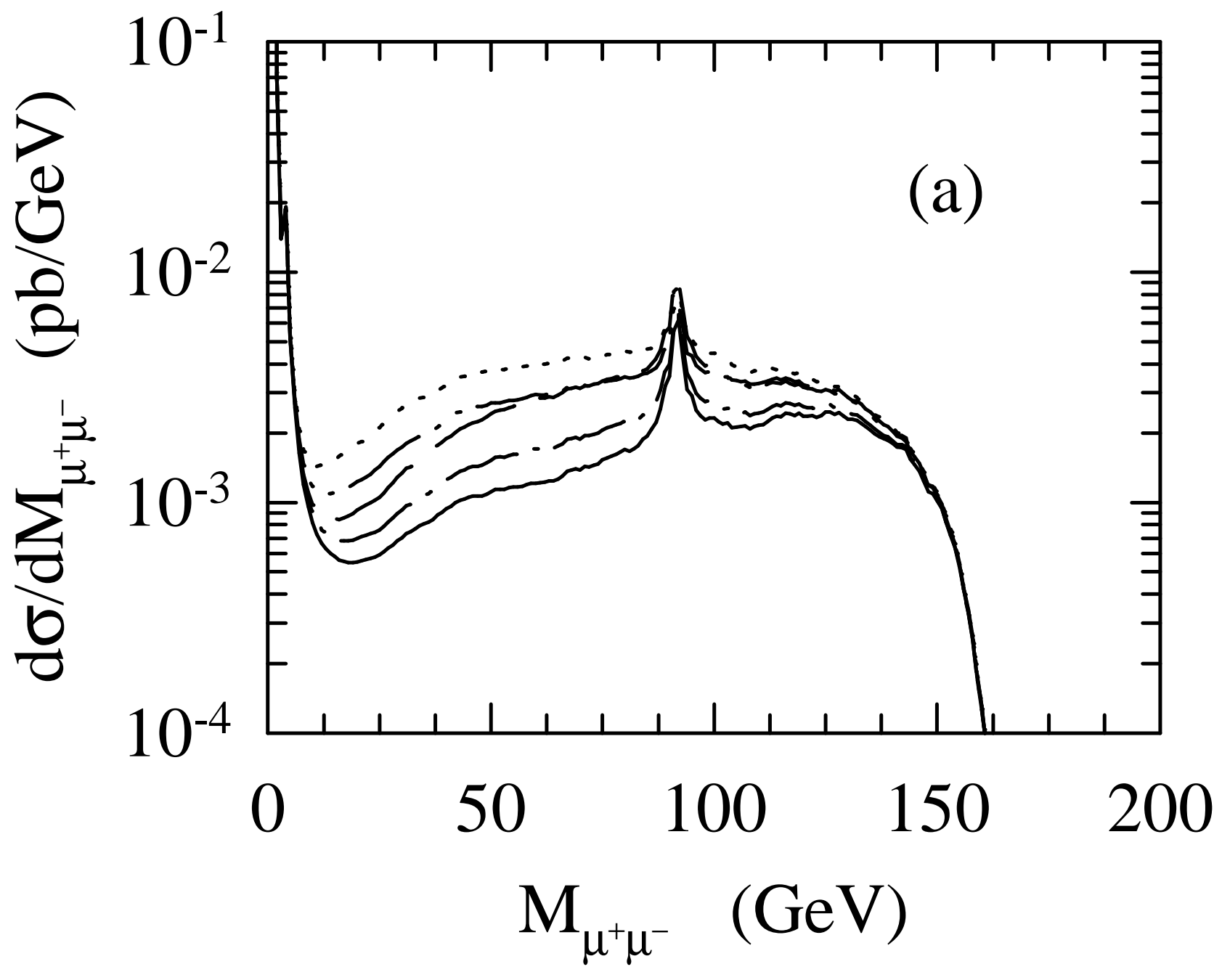




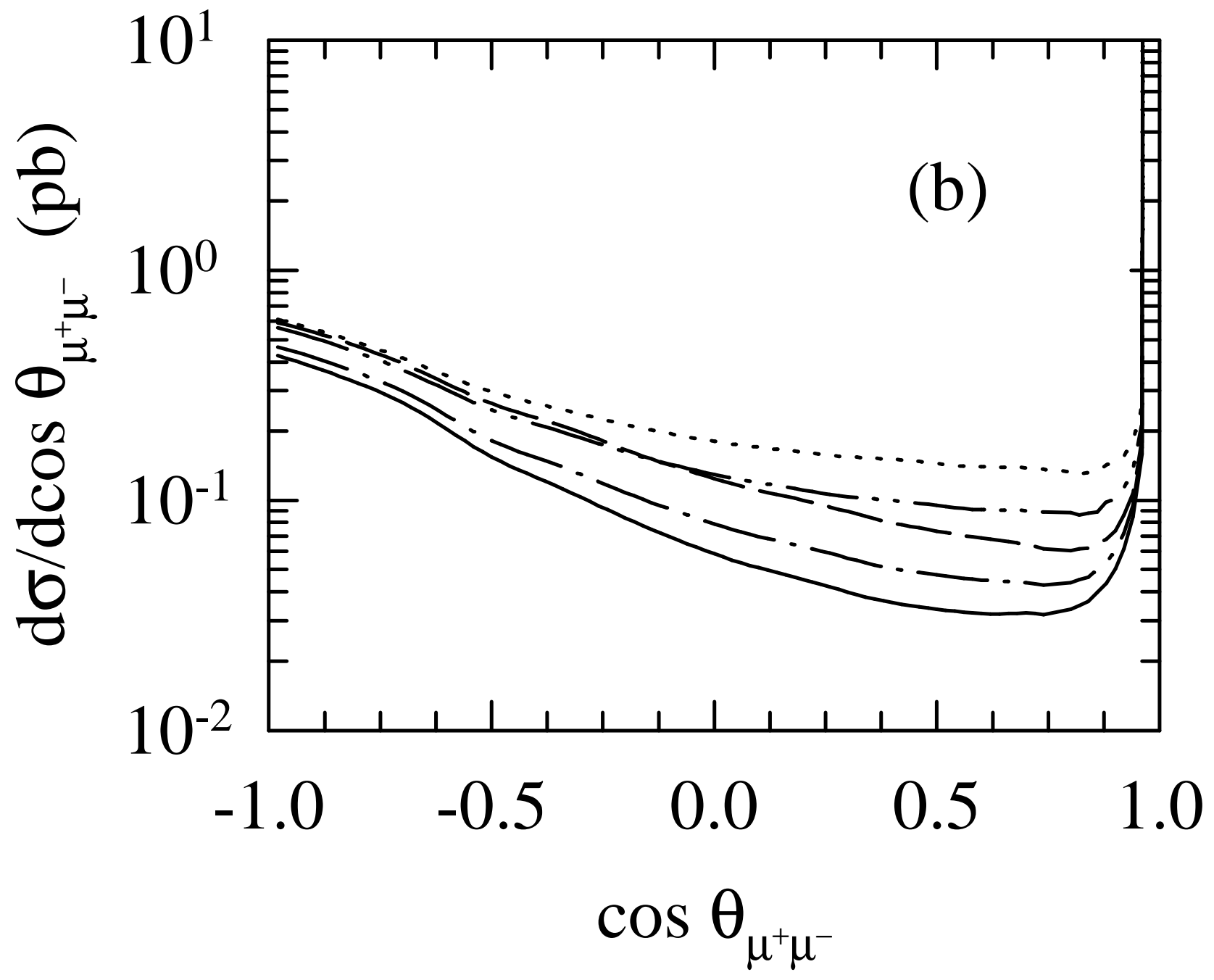


This figure "fig5-4.png" is available in "png" format from: http://arxiv.org/ps/hep-ph/9401306v1 
This figure "fig2-5.png" is available in "png" format from: http://arxiv.org/ps/hep-ph/9401306v1 
This figure "fig3-5.png" is available in "png" format from: http://arxiv.org/ps/hep-ph/9401306v1 
This figure "fig5-5.png" is available in "png" format from: http://arxiv.org/ps/hep-ph/9401306v1 


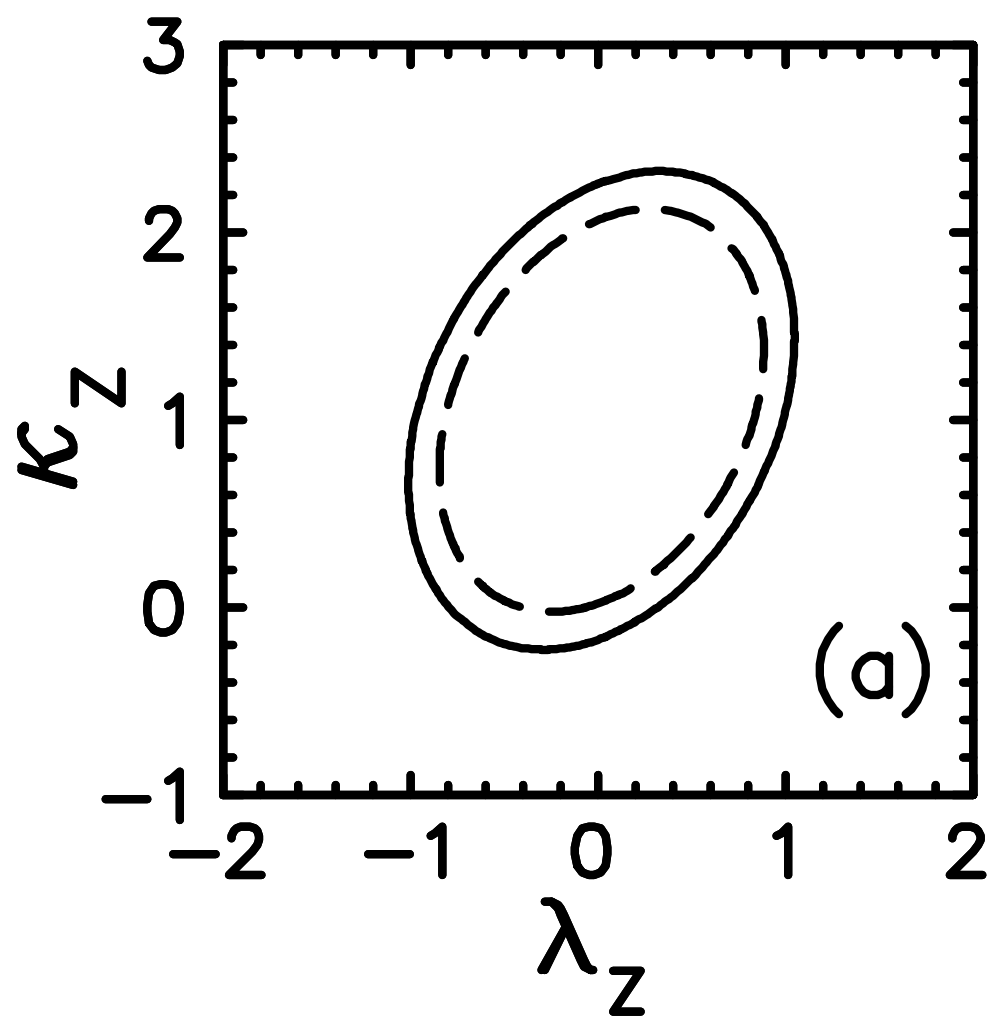




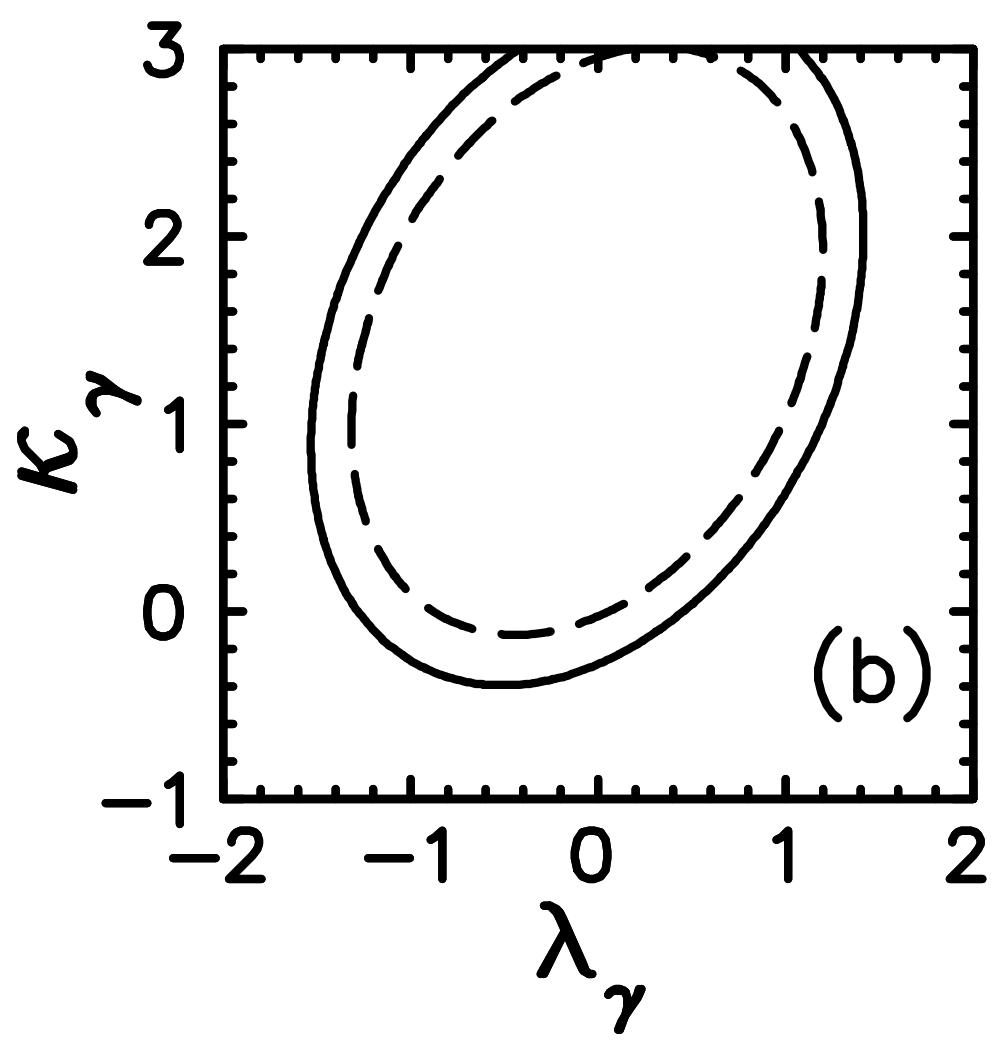




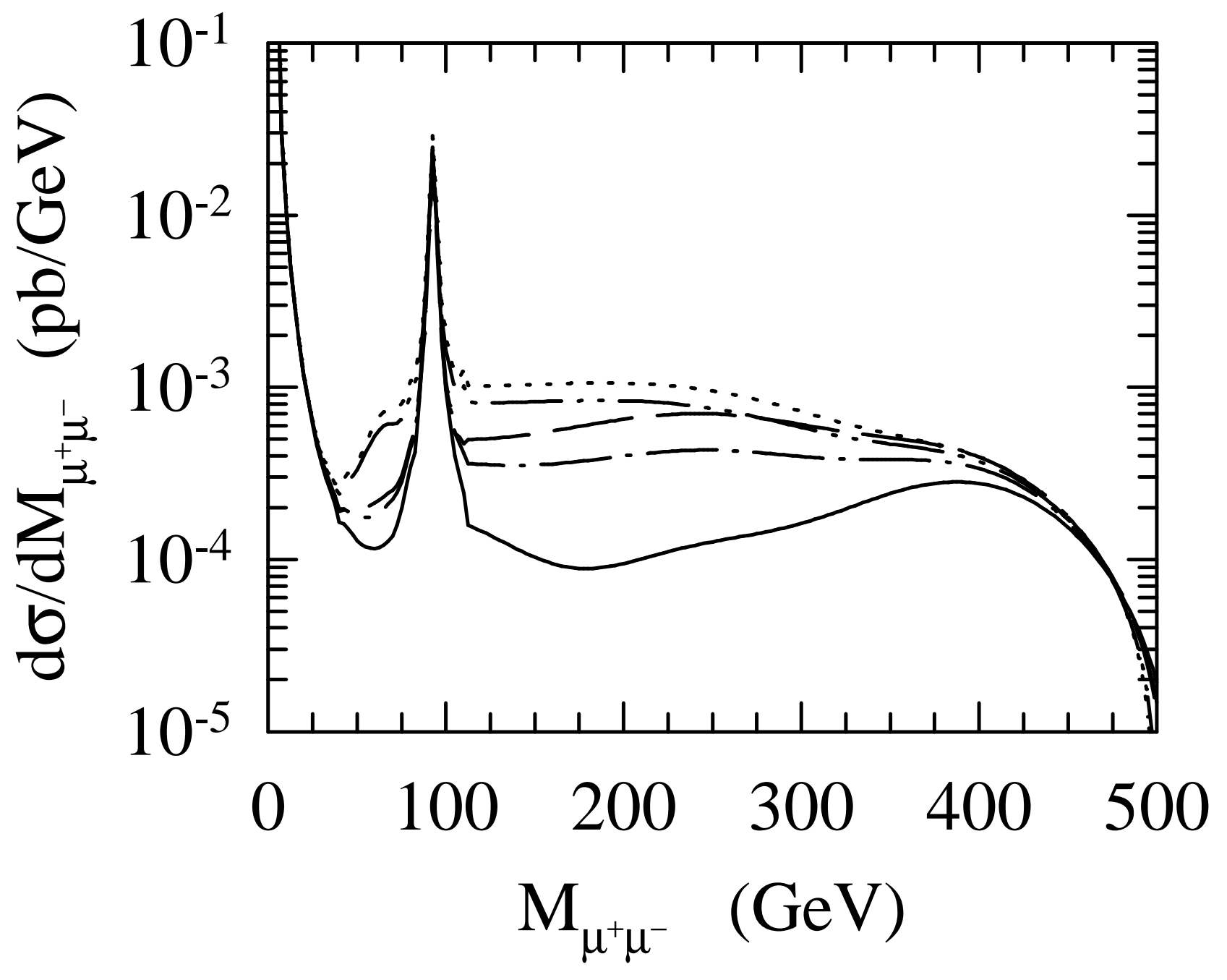




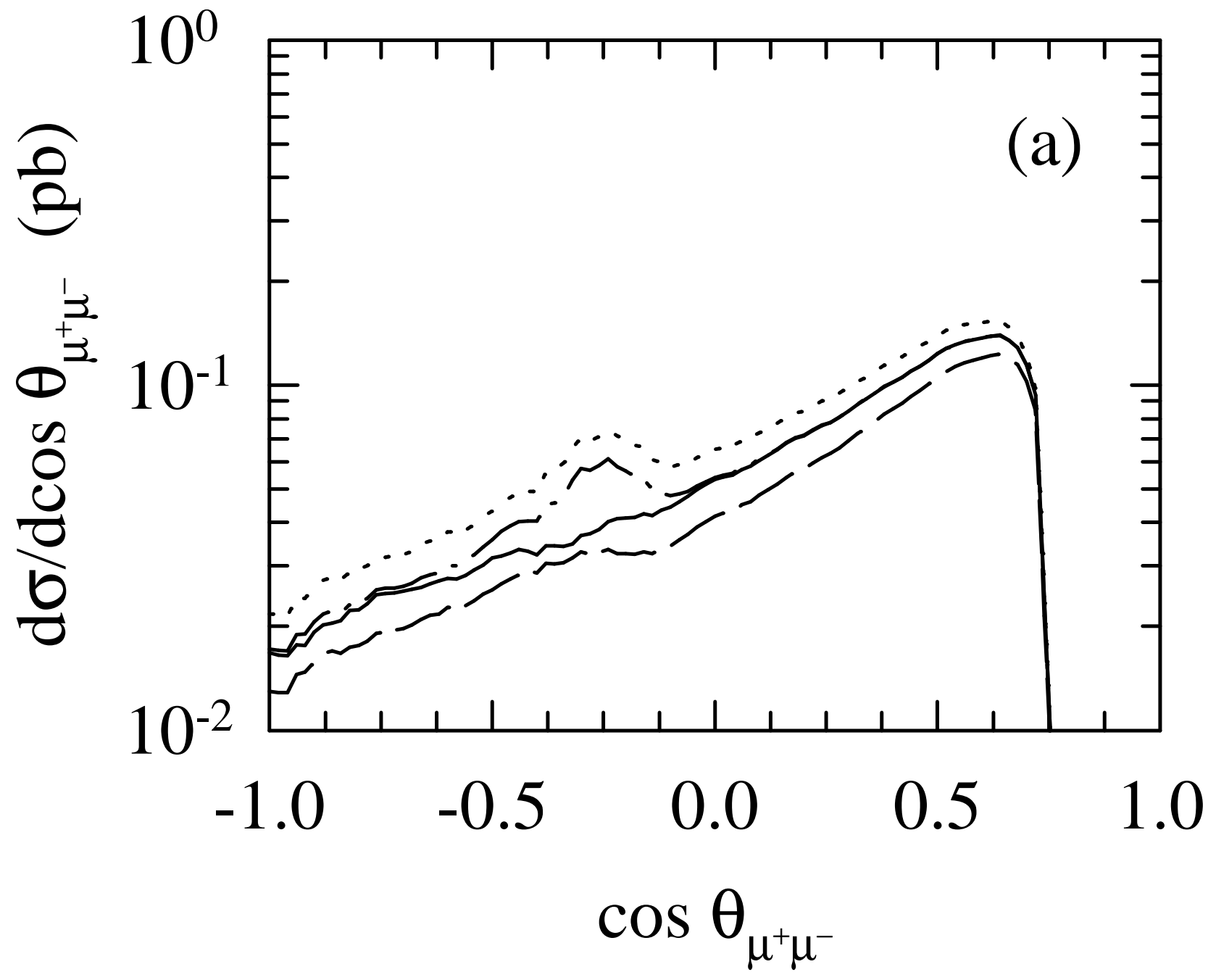




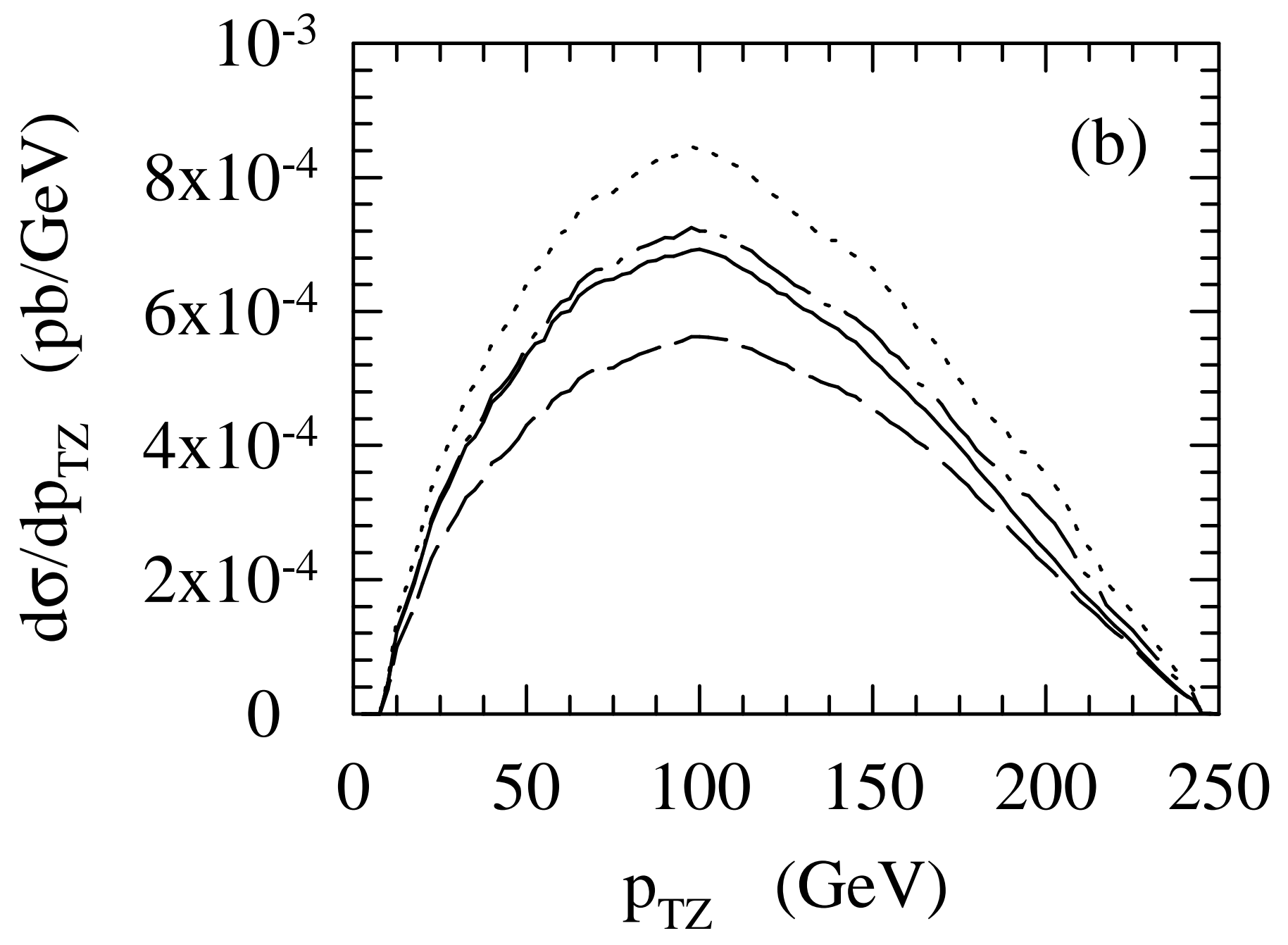




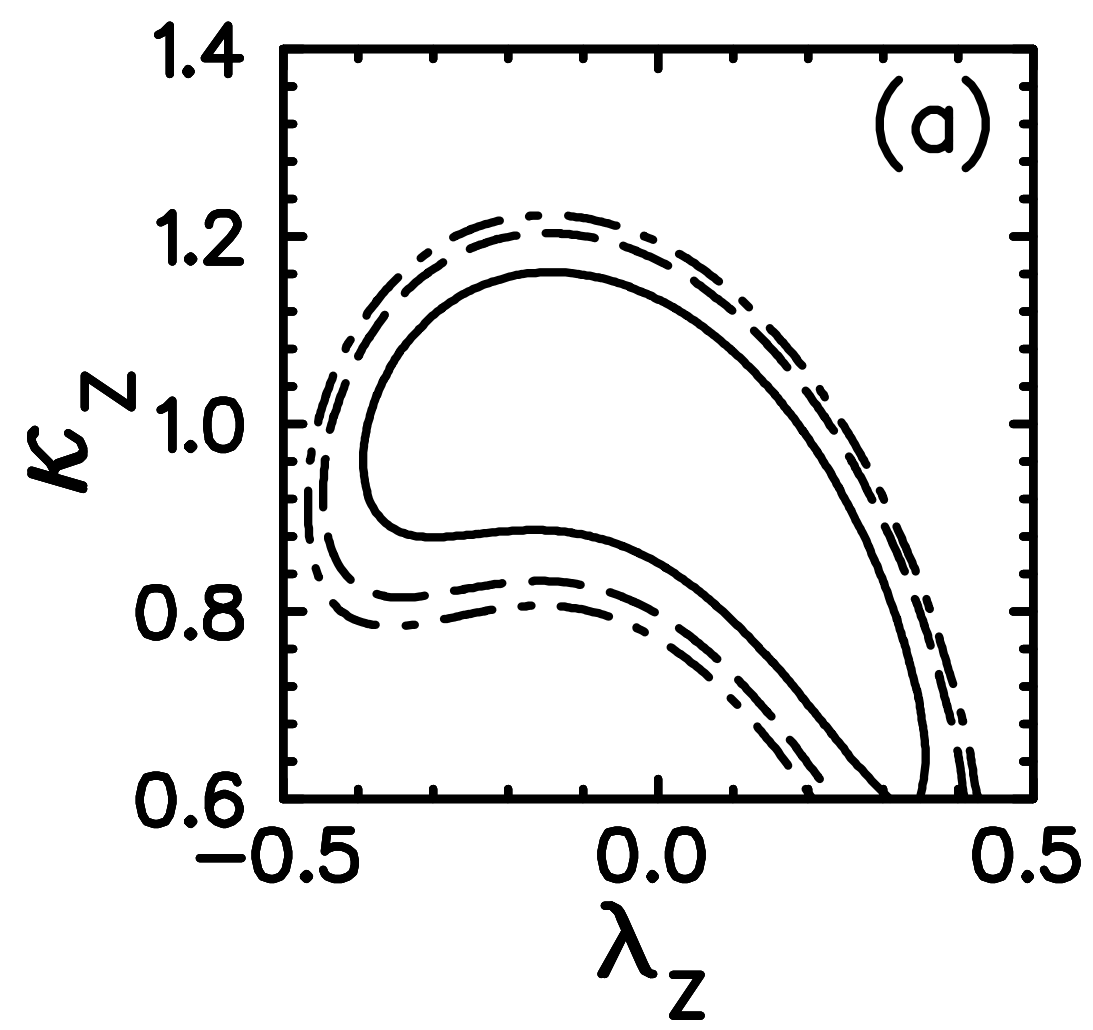




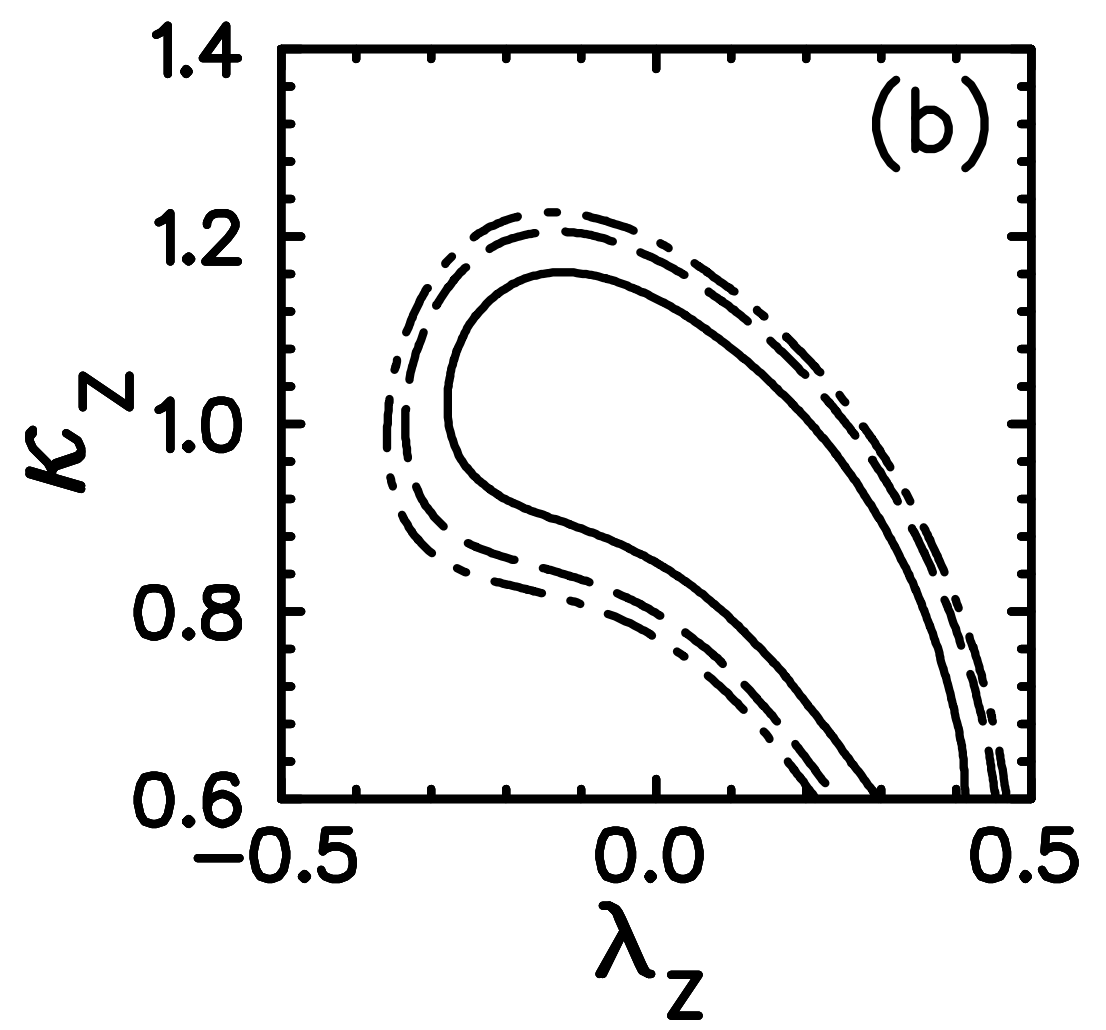




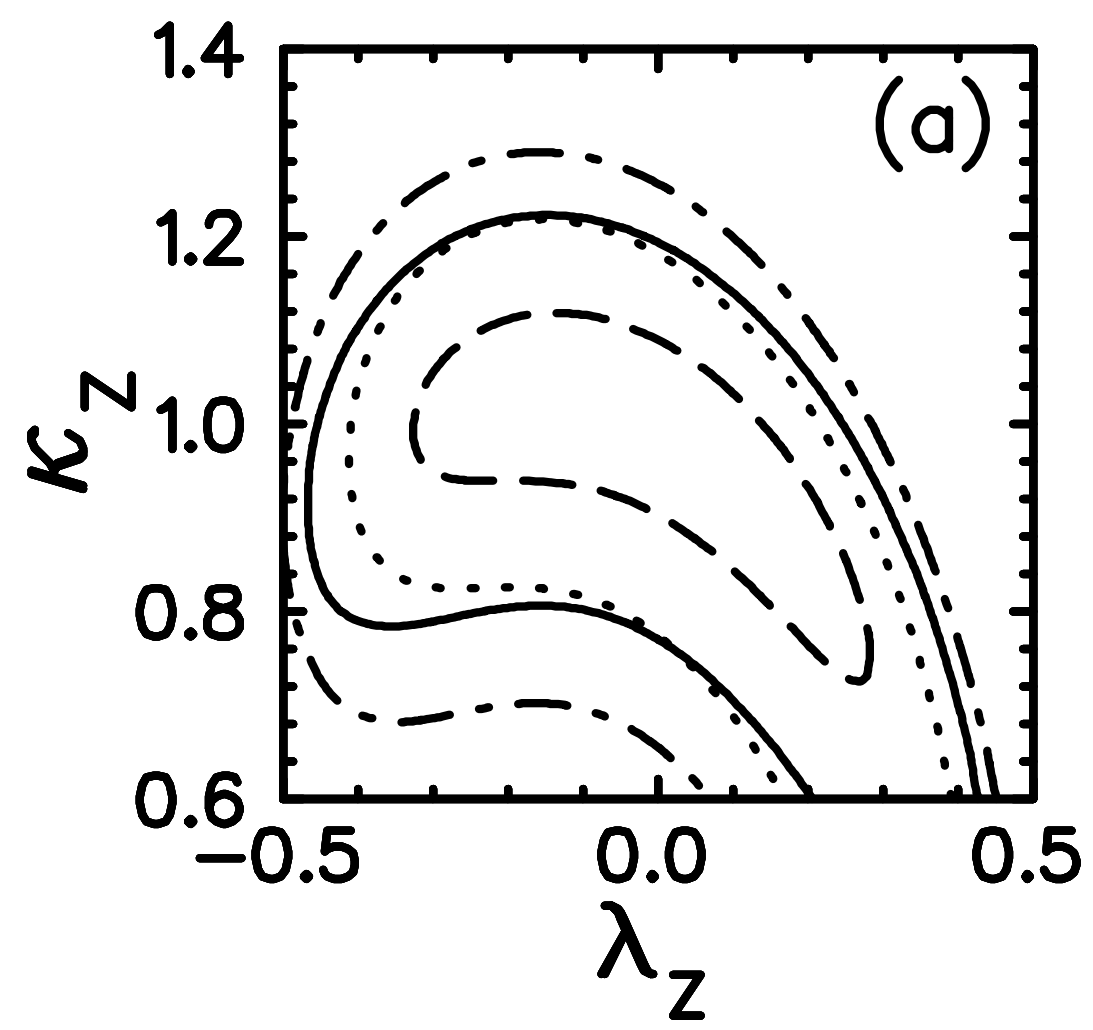




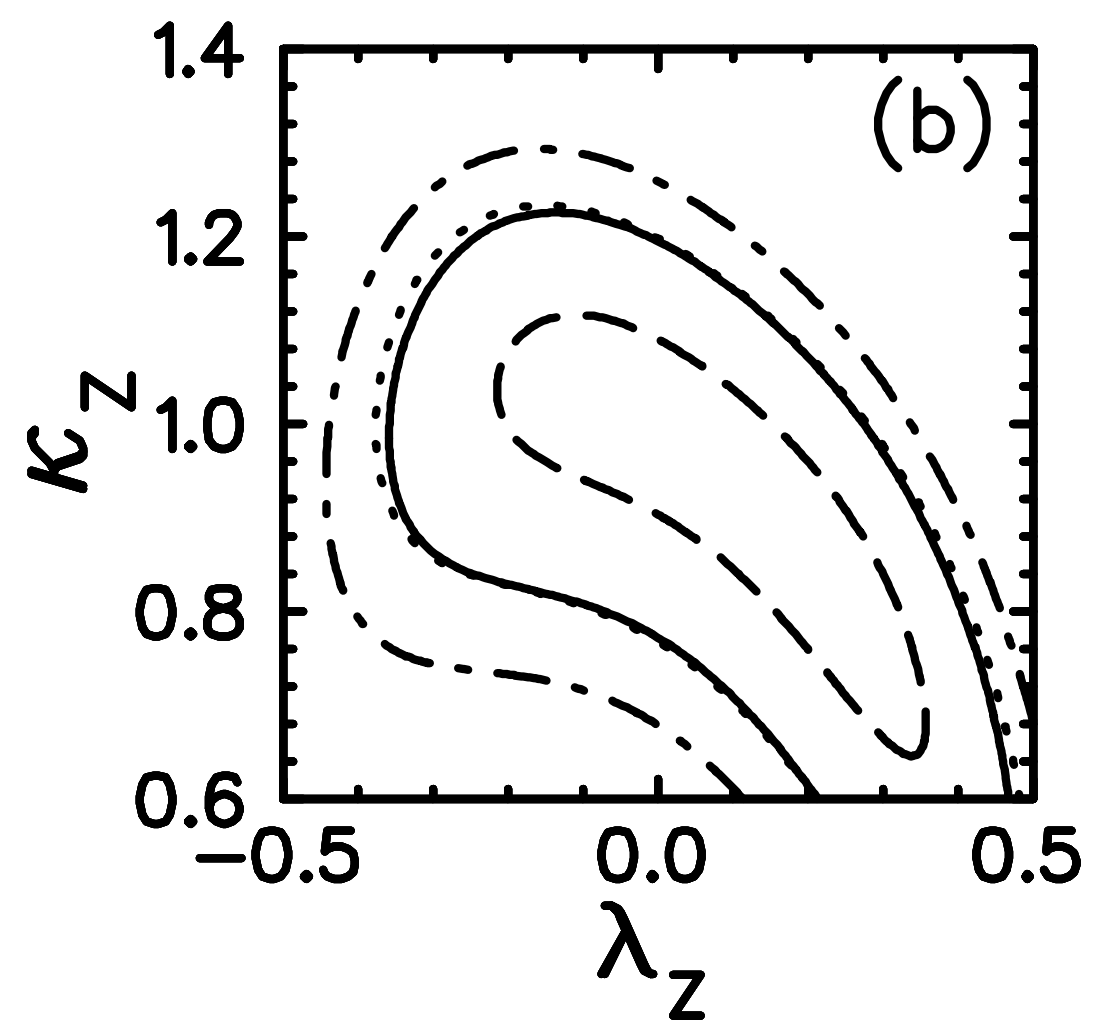




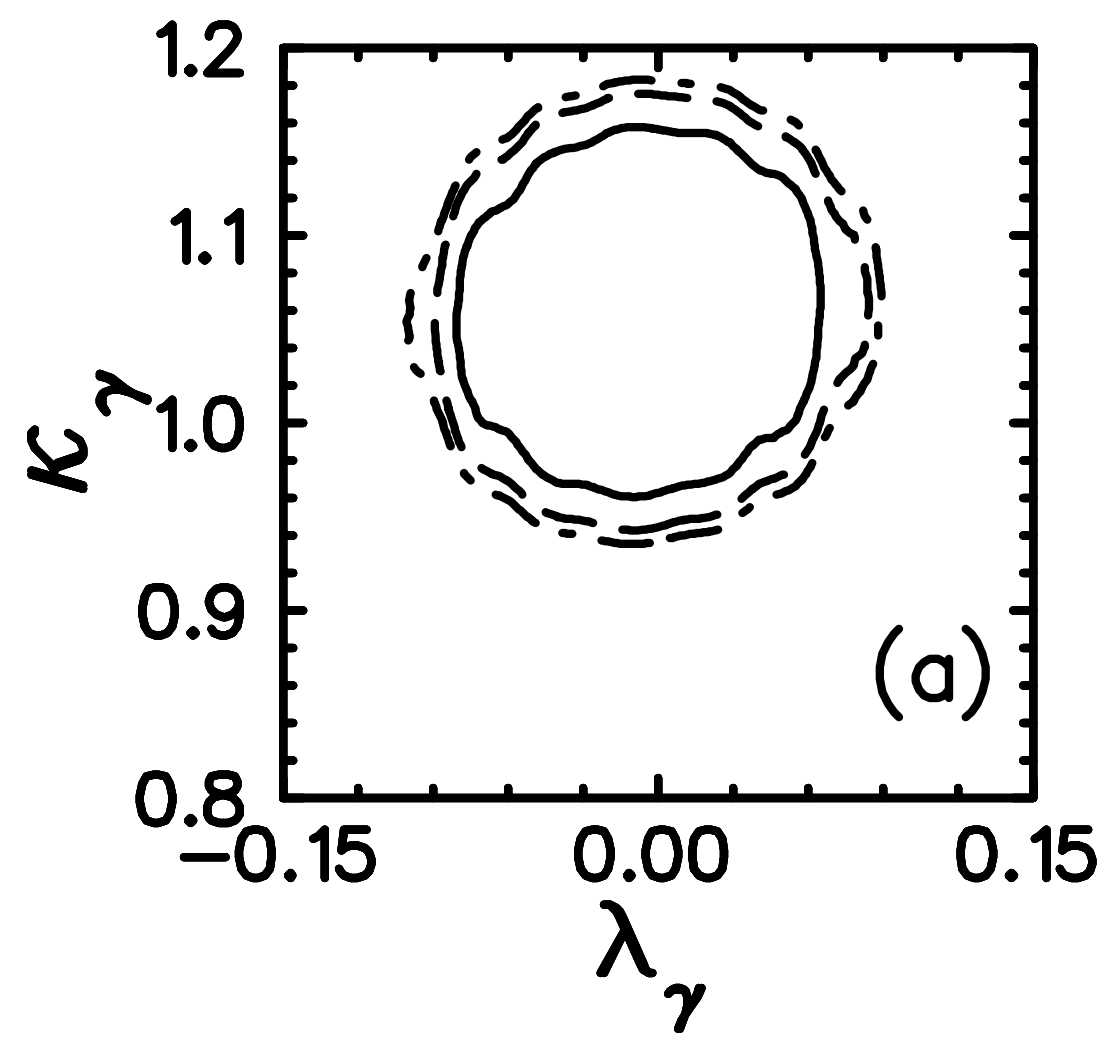




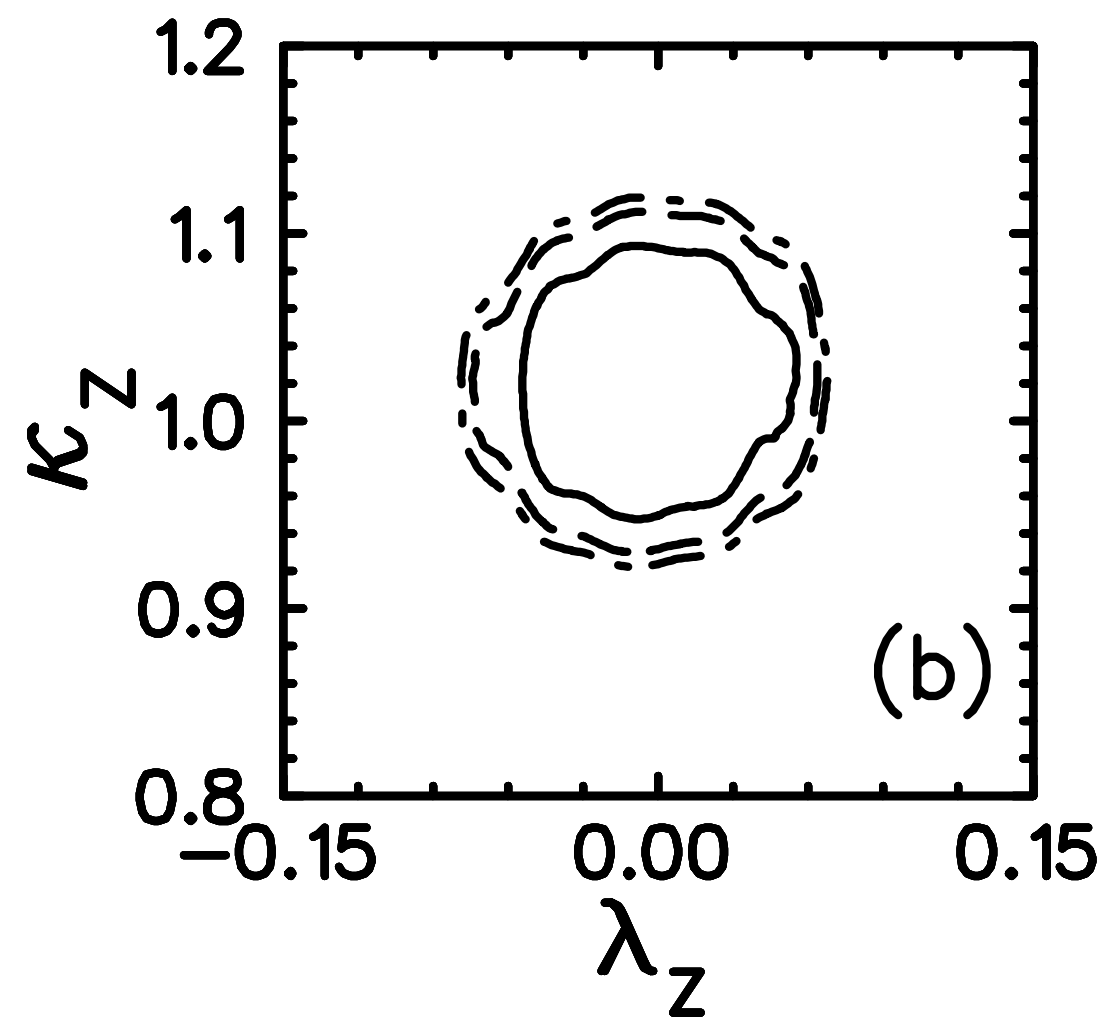




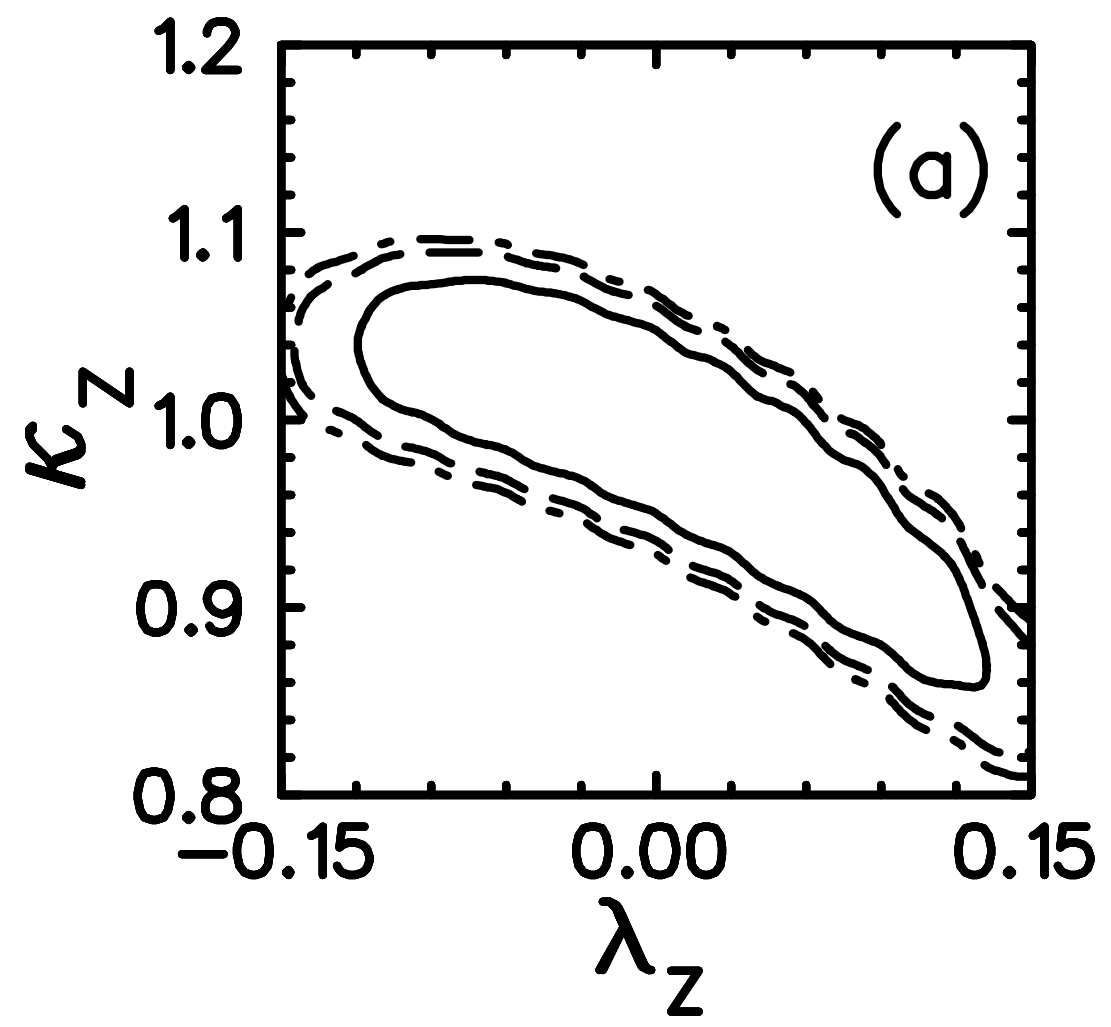




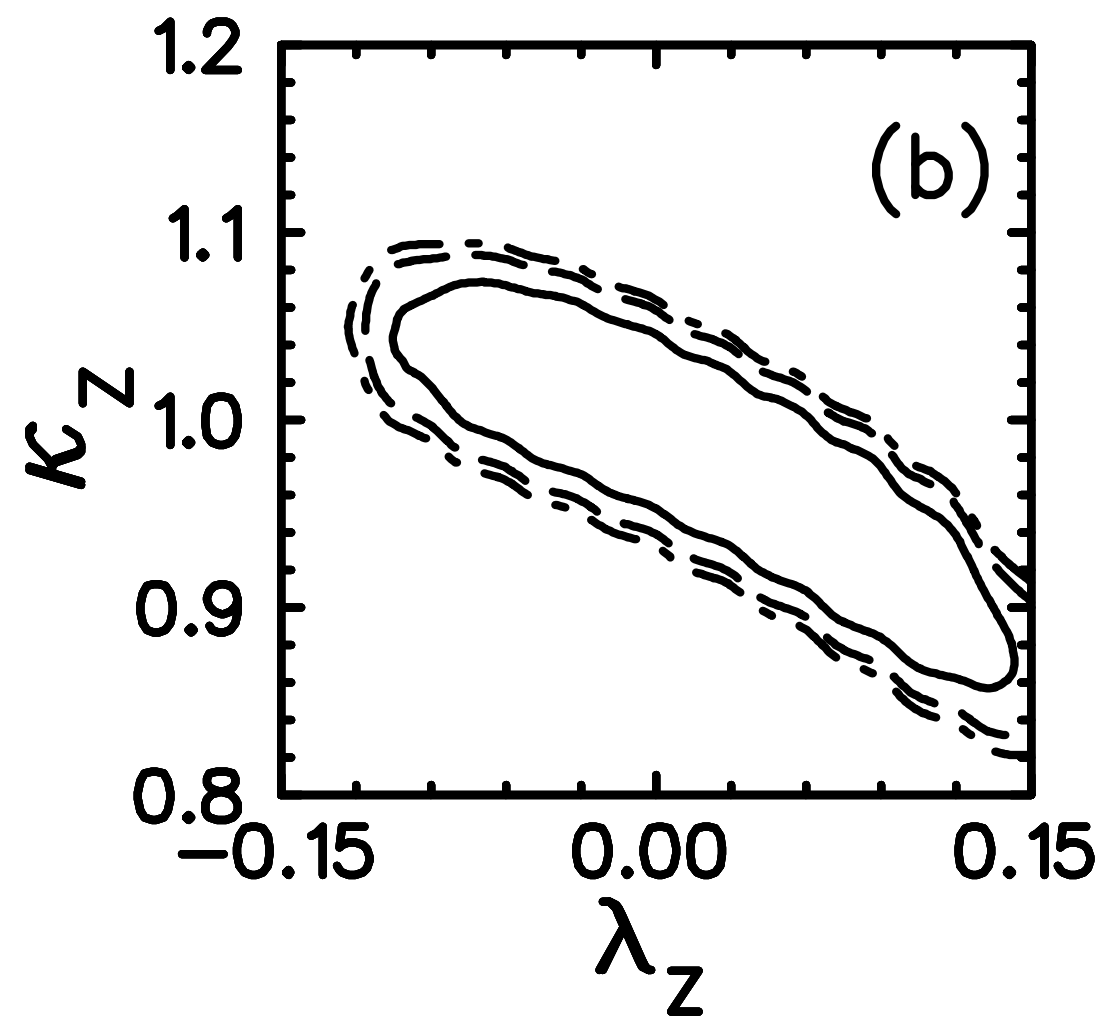




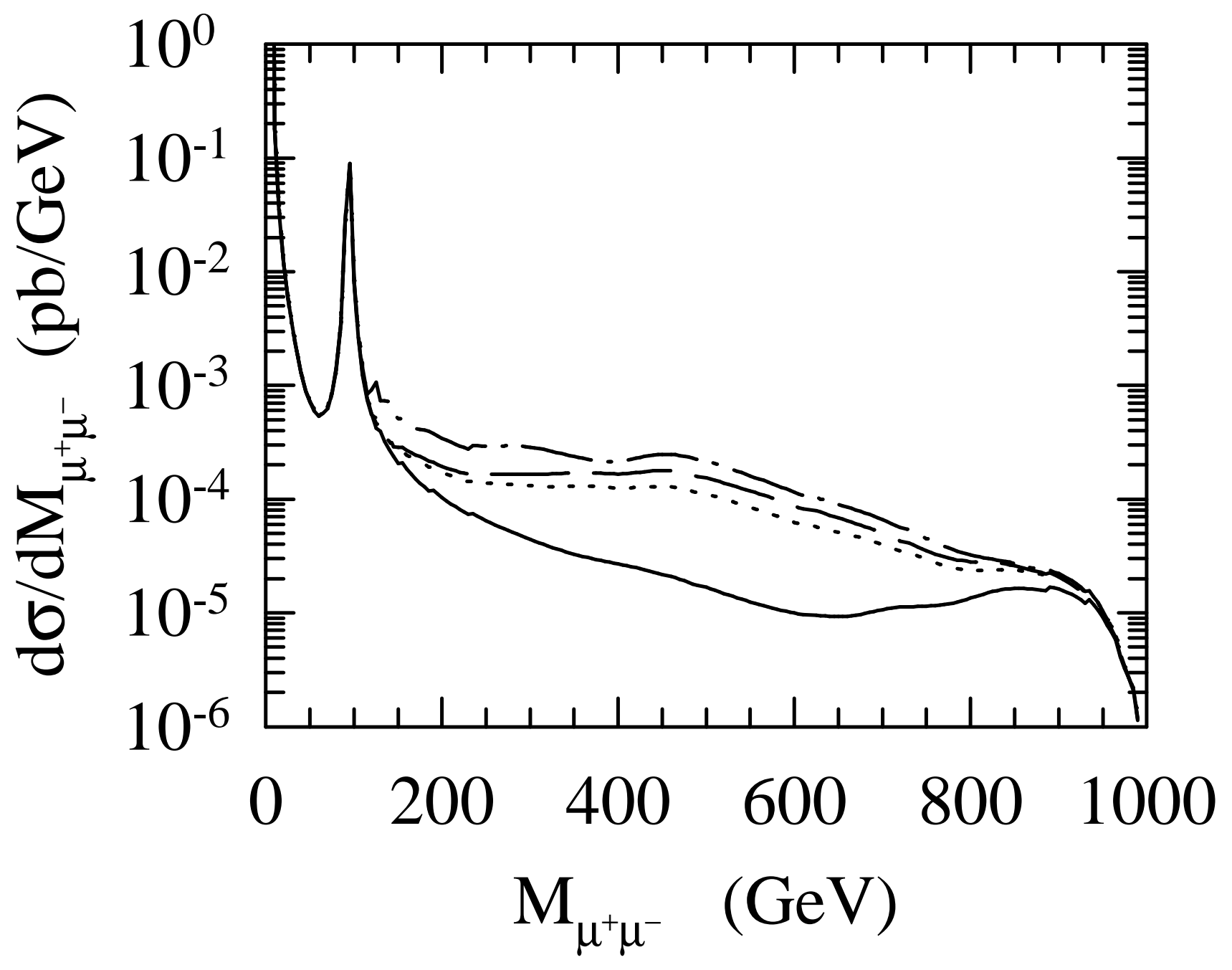




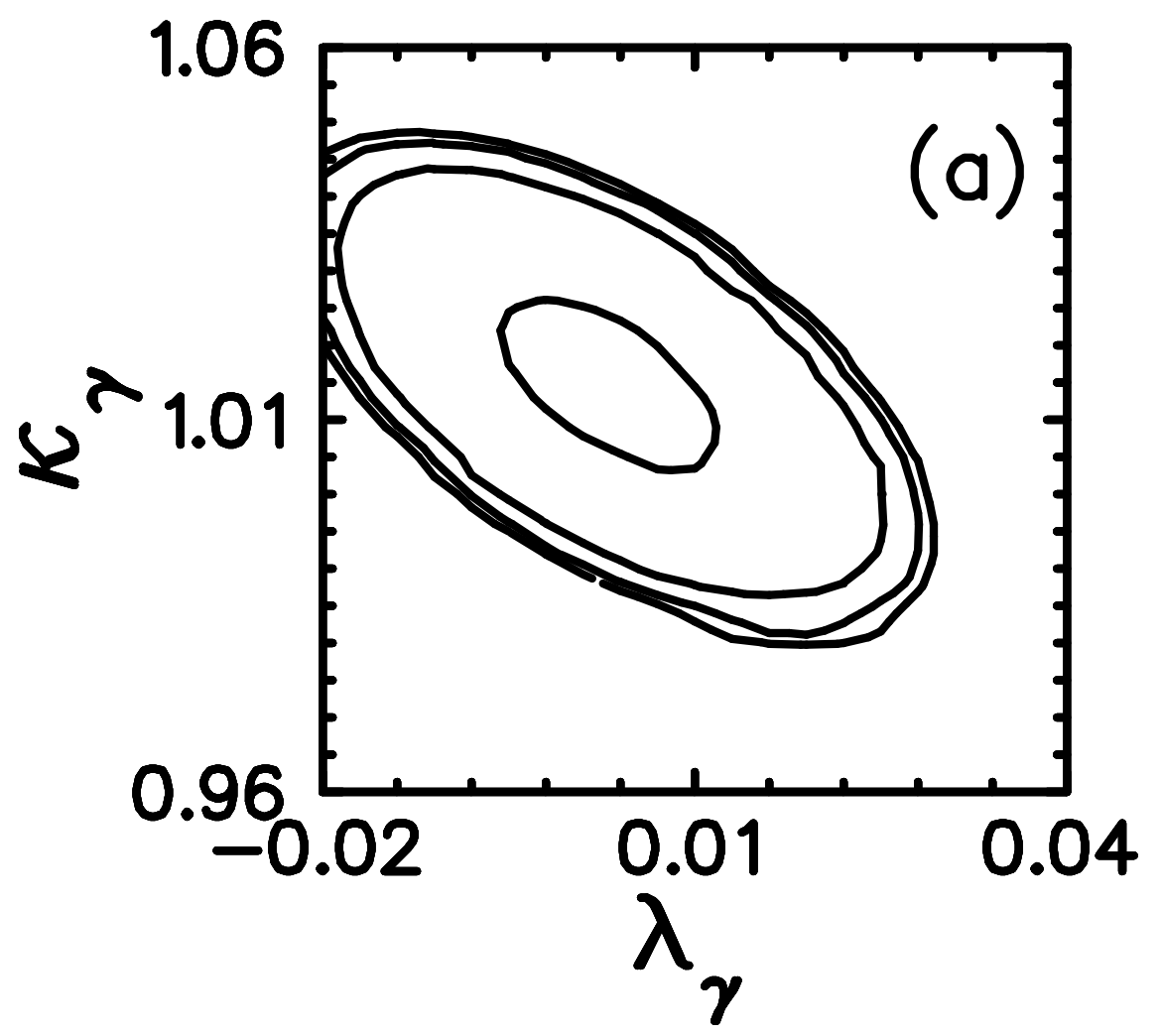




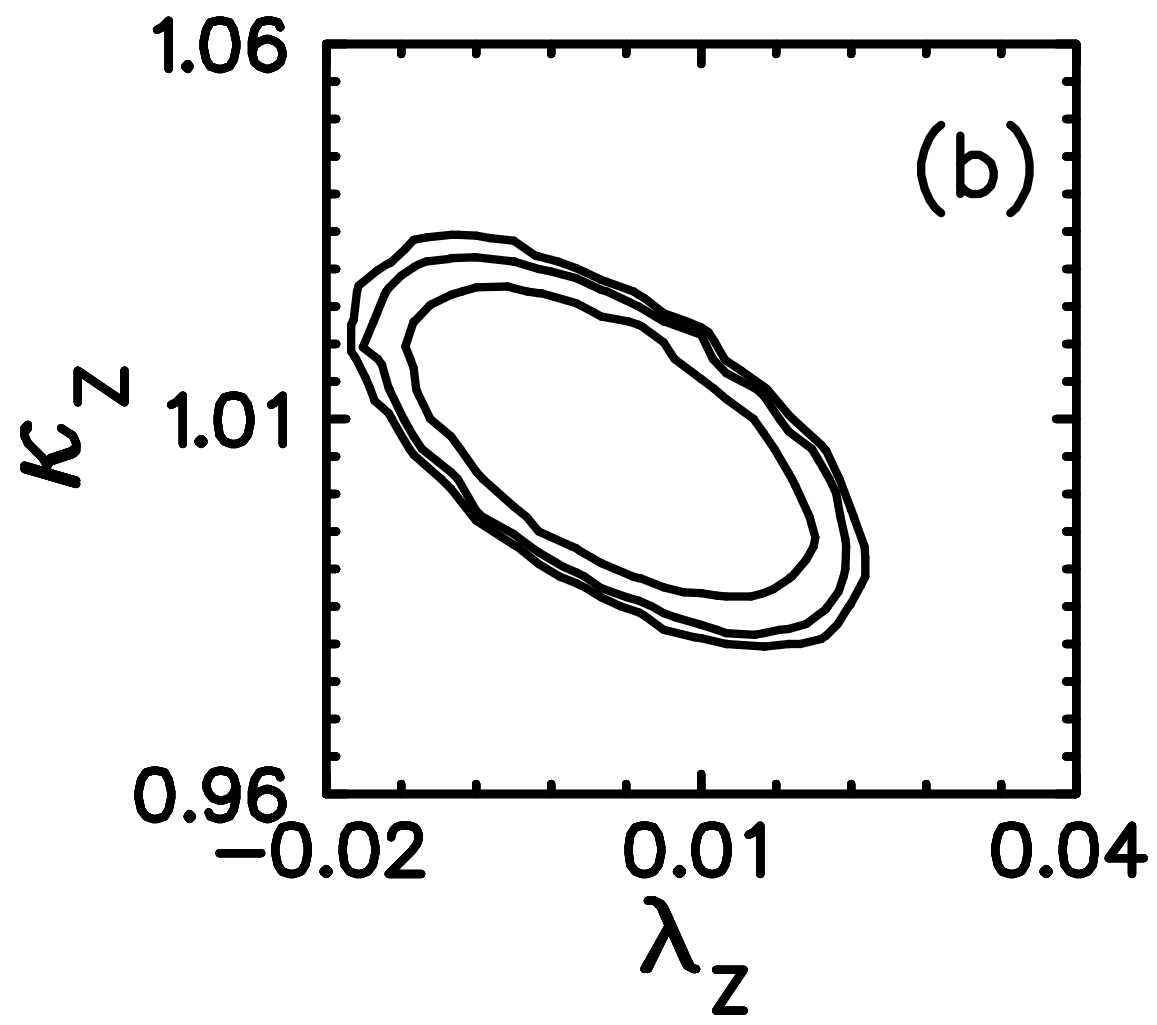

